\title{
New Method of Solving Complicated Operational Amplifier Systems and Application to Online Electrocardiograph
}

\author{
Chung-Cheng Chen, ${ }^{1 *}$ Yen-Ting Chen, ${ }^{2,3}$ and Jian-Hui Li ${ }^{1}$ \\ ${ }^{1}$ City College of Dongguan University of Technology, \\ No. 1, Wenchang Road, Liaobu Town, Dongguan, Guangdong 523419, China \\ ${ }^{2}$ Department of Automatic Control Engineering, Feng Chia University, \\ No. 100, Wenhwa Rd., Seatwen, Taichung, Taiwan 40724, R.O.C. \\ ${ }^{3}$ Department of Electrical Engineering, Graduate School, National Chung Hsing University, \\ 145 Xingda Rd. South Dist., Taichung, Taiwan, R.O.C.
}

(Received February 18, 2019; accepted May 20, 2019)

Keywords: Kirchhoff's law, virtual ground technique, online electrocardiograph, authors' invented adder, instrumentation amplifier

We propose a new systematic matrix approach, which is an alternative to the disordered virtual ground technique, the Kirchhoff's law, and traditional Thevenin approaches, of designing the desired performance and simultaneously finding two Thevenin equivalent parameters for general complicated operational amplifier systems. One cannot apply the virtual ground method to non-ideal and partially ideal operational amplifier systems including instrumentation and frequency sensors. There has been no matrix method for efficiently solving a complicated operational amplifier circuit for conventional tedious approaches that apply Kirchhoff's law. We also propose a new method of finding Thevenin equivalent circuits for general complicated operational amplifier systems that has significant merits over traditional approaches including the open-circuit voltage and short-circuit current methods, the matrix node voltage (mesh current) method, the modified nodal (mesh) analysis, the reference node $r$ model, the reflected impedance method, and the virtual ground method. To compare the proposed and traditional approaches, one demonstrated example and one comparative example are chosen. Moreover, we report a Bessel low-pass filter that is mainly made up of an AD8618 quad op-amp. Its transfer function for existing methods is incorrect in the non-ideal case, and thus the important amplitude-frequency and phase-frequency characteristic curves will not be exact. An online electrocardiograph (ECG) device is an important tool for many industrial medicine applications and is investigated in this study to demonstrate the practical value of the proposed results.

\section{Introduction}

In general, sensors are specifically designed to sense changes in external conditions and convert the changes into numerical values. The AD620 and AD8220 instrumentation

*Corresponding author: e-mail: ccc49827@ms25.hinet.net

https://doi.org/10.18494/SAM.2019.2336 
sensors are used to sense the physiological signal change. Also, the frequency sensors are applied to sense the environmental frequency signal change. The frequency sensors include $60 \mathrm{~Hz}$ notch, low-pass, and high-pass filters. In this study, we propose an innovative design method for complex operational amplifier systems to develop the authors' invented adder, instrumentation amplifiers, notch filters, low-pass filters, and high-pass filters, and to replace the traditional methods of using the disordered virtual ground property and Kirchhoff's voltage law (Kirchhoff's current law). In particular, for more complicated interconnected operational amplifier systems with some dependent elements, it is difficult for traditional approaches to solve the desired function via Kirchhoff's voltage law (Kirchhoff's current law). To compare the proposed complex operational amplifier method with traditional methods, a comparative example is chosen to illustrate the theoretical points in detail. Note that up to now, there has been no matrix method for efficiently solving complicated operational amplifier circuits using conventional approaches. We also report a Bessel low-pass filter that is mainly made up of an AD8618 quad op-amp for comparison with other existing methods, ${ }^{(14)}$ as a practical example in Sect. 3. The transfer function of the filter shown in Ref. 14 is incorrect in the non-ideal case. Moreover, the transfer functions of the AD8220 instrumentation and main AD8618 amplifiers are incorrect in the non-ideal case, and thus the important amplitude-frequency and phasefrequency characteristic curves will not be exact.

An operational amplifier is a DC-coupled high-gain electric device with a differential input pair and a single-ended output. ${ }^{(23)}$ Operational amplifiers have important applications in analog computers, where they are used to implement mathematical operations in many industrial circuits. However, research using the virtual ground technique and Kirchhoff's voltage law (Kirchhoff's current law) shows that for more complex operational amplifier systems containing dependent sources and coupling elements, the derivations of the four amplifier performance indexes, i.e., the Thevenin equivalent circuit, voltage gain, current gain, and equivalent resistance, become rather tedious. ${ }^{(1)}$ Moreover, we cannot apply the virtual ground property in non-ideal operational amplifier systems. To overcome these shortcomings, a simple and systematic unifying approach is proposed in this study to extend our previous results in Refs. 3-5 to solve a complex operational amplifier.

The existing conventional approaches to designing the desired performance for general complicated operational amplifier systems are summarized as follows:

(Method 1): Obtain the desired performance for general complicated operational amplifier systems based on the virtual ground method. However, one cannot apply the virtual ground method to non-ideal and partially ideal operational amplifier systems. ${ }^{(20,21)}$

(Method 2): Obtain the desired performance for general complicated operational amplifier systems via Kirchhoff's law. However, no matrix method has been proposed for efficiently solving a complicated operational amplifier circuit for conventional approaches that apply the tedious Kirchhoff's law. ${ }^{(17)}$

(Method 3): Apply the Thevenin equivalent theorem to design the desired performance. ${ }^{(10,22)}$ The conventional standard methods of deriving the Thevenin equivalent circuit involve two steps as follows: Step 1: Let the load device be open-circuited and the Thevenin voltage parameter be calculated as the open-circuit voltage across the load device. As can be 
clearly seen in Refs. 1 and 17, the calculation of the open-circuit voltage using Kirchhoff's voltage law (Kirchhoff's current law) is tedious when the network has complicated devices with dependent sources and coupling devices. Step 2: The remaining task of finding the Thevenin equivalent circuit requires the derivation of the Thevenin resistance parameter. There are three main techniques for deriving the Thevenin resistance parameter used in recent research as follows. Technique 1: Set all independent sources to zero with the load device disconnected. Then, the equivalent resistance of the zero-energy circuit at the load device is the desired Thevenin resistance parameter obtained via the conventional nodevoltage matrix method. ${ }^{(7)}$ Technique 1 cannot be utilized when a voltage (current) source is not in series (parallel) with a resistor owing to the intrinsic properties of the conventional node-voltage (mesh-current) matrix method. Technique 2: Find the open-circuit voltage $V_{O C}$ and short-circuit current $I_{S C}$ across the load device, then the Thevenin resistance parameter is equal to $Z_{T H}=V_{O C} I_{S C} .^{(8,17)}$ Technique 2 is not only impractical but also has the risk of encountering the indeterminate case of $0 / 0$. For some circuits consisting of only dependent sources and resistors, one needs to apply an alternative method because the ratio of $V_{O C}$ to $I_{S C}$ has the indeterminate form 0/0. Technique 3: Firstly, set all independent sources to zero with the load device disconnected. Then, apply an additional independent current (voltage) source $I_{t}\left(V_{t}\right)$ to the load device, then find the load voltage (current) $V_{k}\left(I_{k}\right)$ using $Z_{T H}=V_{k} / I_{t}$ $\left(Z_{T H}=V_{t} / I_{k}\right) .{ }^{(1)}$ Technique 3 is restricted to certain conditions owing to the requirement of setting all independent sources to zero. Moreover, it is also obvious that using conventional Technique 3 with Kirchhoff's voltage law (Kirchhoff's current law) is very tedious.

To solve all the above shortcomings of the conventional approaches, we propose an efficient method of designing the desired performance for general complicated operational amplifier systems in a straightforward manner. The significant novel features of this proposed approach includes the following. (1) It can easily be applied to electrical operational amplifier circuits in which the voltage (current) source is not in series (parallel) with a resistor or a dependent source element without using tedious Kirchhoff's law. (2) In this paper, we also propose a new method of finding the Thevenin equivalent circuits for general complicated operational amplifier systems that has systematic solving processes over traditional approaches including the open-circuit voltage and short-circuit current methods, the matrix node voltage (mesh current) method, the modified nodal (mesh) analysis, the reference node $\mathrm{r}$ model, the reflected impedance method, and the virtual ground method. (3) This model is applicable to both nonideal and partially ideal operational amplifier systems. (4) It is conceptually simpler than other methods since it uses systematic matrix operations.

An online electrocardiograph (ECG) device is a diagnostic instrument that is generally utilized to evaluate the electrical and muscular performance characteristics of the heart. ${ }^{(12)}$ The interpretation of ECG traces needs a considerable amount of training. The heart is basically a two-stage electrical pump and its electrical behavior can be gauged by electrodes located on the skin. An ECG can gauge the frequency and rhythm of the heartbeat, as well as indirectly show the blood flow to the heart muscle. A standardized model has been created for the electrode placement of an ECG. A heart monitor generally requires three electrode leads that are on the left chest, left arm, and right arm. The signals collected from each electrode are summarized and printed as an electrocardiograph. 
The heart is the pump responsible for blood circulation, and cardiovascular disease has become the most severe disease affecting human health worldwide. The diagnosis and treatment of cardiovascular disease is a major medical problem. An ECG containing a biological electric signal with amplitude, frequency, and phase is important for clarifying heart activity. The ECG signal shows the working status of the heart from different viewpoints using fundamental body physiological parameters. Measuring these physiological parameters has high clinical significance for the treatment of cardiovascular disease. ${ }^{(13)}$ The ECG thus plays an important role in the clinical treatment of cardiovascular disease. Our online electrocardiograph device has been designed with a single chip and has high performance and low power consumption. The system utilizes high-performance AD620 instrumentation amplifier chips together with an AD0804 A/D chip to implement ECG measurement and communication conveniently. The proposed online monitoring system has the advantages of simple structure, low power consumption, high performance, and easy use. An online electrocardiograph device is an important tool for many industrial medicine applications and our proposed system is investigated in this study to demonstrate the practical application of the proposed results.

\section{New Method of Solving Complicated Operational Amplifier Systems.}

The newly proposed method of solving complicated operational amplifier systems is demonstrated using the example shown in Fig. 1. By utilizing the L'Hospital rule and the simple matrix operations developed by the authors, an innovative complicated operational amplifier approach is proposed as follows:

(Step 1) Draw the s-domain operational amplifier equivalent circuit of Fig. 1 and use a technique to transform all voltage sources to current sources, where $R_{1}=k R, R_{2}=2 k R$. If the voltage source is not in series with a resistor, we can place a zero resistor $R_{c c c}$ in series with the voltage source, and thus the matrix node voltage method can be applied.

(Remark 1) The useful technique of adding a zero resistor $R_{c c c}$ has not been proposed in research on the traditional matrix node voltage method.

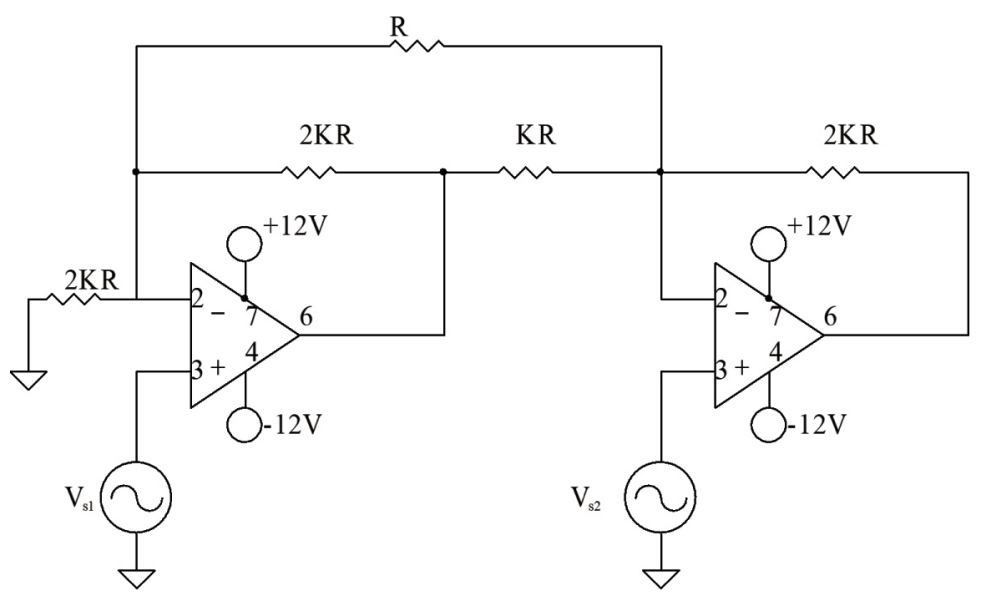

Fig. 1. Demonstrated example of solving complicated operational amplifier system. 
(Step 2) List the node-voltage matrix equations in the following format:

$$
\left[\begin{array}{cccc}
Y_{11}(s) & -Y_{12}(s) & \cdots & -Y_{1 N}(s) \\
-Y_{21}(s) & Y_{22}(s) & \cdots & -Y_{2 N}(s) \\
\vdots & \vdots & & \vdots \\
-Y_{N 1}(s) & -Y_{N 2}(s) & \cdots & Y_{N N}(s)
\end{array}\right]\left[\begin{array}{c}
V_{1}(s) \\
V_{2}(s) \\
\vdots \\
V_{N}(s)
\end{array}\right]=\left[\begin{array}{c} 
\pm I_{S 11}(s) \pm I_{S 12}(s) \pm \cdots \\
\pm I_{S 21}(s) \pm I_{S 22}(s) \pm \cdots \\
\vdots \\
\pm I_{S N 1}(s) \pm I_{S N 2}(s) \pm \cdots
\end{array}\right]
$$

where associated with $V_{i}, i=1,2, \cdots, N$, are called the node voltages. The node-voltage matrix equations of Fig. 2 are given as

$$
\left[\begin{array}{cccc}
\frac{1}{R_{2}}+\frac{1}{R_{i 1}}+\frac{1}{R_{1}}+\frac{1}{R} & -\frac{1}{R_{1}} & -\frac{1}{R} & 0 \\
-\frac{1}{R_{1}} & \frac{2}{R_{1}}+\frac{1}{R_{o 1}} & -\frac{1}{R_{1}} & 0 \\
-\frac{1}{R} & -\frac{1}{R_{1}} & \frac{1}{R}+\frac{1}{R_{1}}+\frac{1}{R_{i 2}}+\frac{1}{R_{2}} & -\frac{1}{R_{2}} \\
0 & 0 & -\frac{1}{R_{2}} & \frac{1}{R_{2}}+\frac{1}{R_{o 2}}
\end{array}\right]\left[\begin{array}{c}
v_{k 1} \\
v_{k 2} \\
v_{k 3} \\
v_{o}
\end{array}\right]=\left[\begin{array}{c}
\frac{v_{s 1}}{R_{i 1}} \\
\frac{A_{v 1} v_{i 1}}{R_{o 1}} \\
\frac{v_{s 2}}{R_{i 2}} \\
\frac{A_{v 2} v_{i 2}}{R_{o 2}}
\end{array}\right]
$$

(Step 3) Apply the L'Hospital rule to reduce the $R_{c c c}$ terms. Since Fig. 2 does not have additional $R_{c c c}$ terms, this step can be omitted here.

(Step 4) If the dependent current (voltage) variables are involved in the node-voltage matrix equations, the dependent variables must be transformed to node-voltage variables and moving-term operations are immediately executed. In general, an electrical system with a dependent source will have increased complexity in network analysis..$^{(1,17,19)}$ On the other hand, this problem is easily solved by our proposed approach based on the moving-

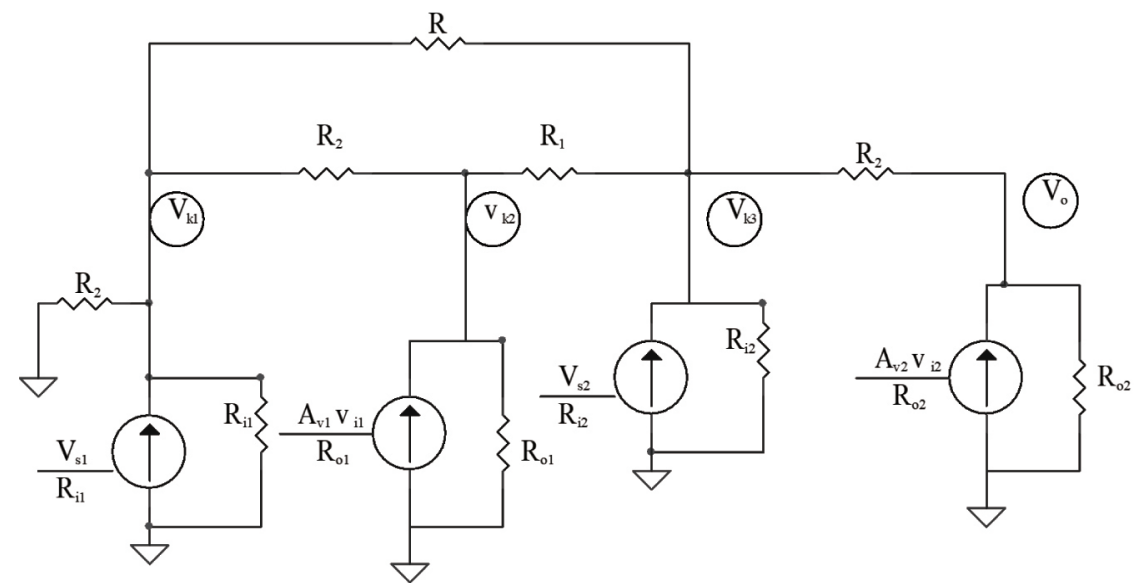

Fig. 2. Equivalent circuit of Fig. 1. 
term operations in this study. Substituting $v_{i 1}=v_{k 1}-v_{s 1}$ and $v_{i 2}=v_{k 3}-v_{s 2}$ into Eq. (2) and moving the node-voltage variables to the other side of the equation yield the following expression:

$$
\left[\begin{array}{cccc}
\frac{1}{R_{2}}+\frac{1}{R_{i 1}}+\frac{1}{R_{1}}+\frac{1}{R} & -\frac{1}{R_{1}} & -\frac{1}{R} & 0 \\
-\frac{1}{R_{1}}-\frac{A_{v 1}}{R_{o 1}} & \frac{2}{R_{1}}+\frac{1}{R_{o 1}} & -\frac{1}{R_{1}} & 0 \\
-\frac{1}{R} & -\frac{1}{R_{1}} & \frac{1}{R}+\frac{1}{R_{1}}+\frac{1}{R_{i 2}}+\frac{1}{R_{2}} & -\frac{1}{R_{2}} \\
0 & 0 & -\frac{1}{R_{2}}-\frac{A_{v 2}}{R_{o 2}} & \frac{1}{R_{2}}+\frac{1}{R_{o 2}}
\end{array}\right]\left[\begin{array}{c}
v_{k 1} \\
v_{k 2} \\
v_{k 3} \\
v_{o}
\end{array}\right]=\left[\begin{array}{c}
\frac{v_{s 1}}{R_{i 1}} \\
-\frac{A_{v 1} v_{s 1}}{R_{o 1}} \\
\frac{v_{s 2}}{R_{i 2}} \\
-\frac{A_{v 2} v_{s 2}}{R_{o 2}}
\end{array}\right]
$$

(Step 5) Applying the special characteristics $R_{i}=\infty, R_{o}=0$, and $A_{v}=-\infty$ of the ideal operational amplifier reduces the equation to

$$
\left[\begin{array}{cccc}
\frac{1}{R_{2}}+0+\frac{1}{R_{1}}+\frac{1}{R} & -\frac{1}{R_{1}} & -\frac{1}{R} & 0 \\
0-\frac{A_{v 1}}{R_{o 1}} & 0+\frac{1}{R_{o 1}} & -\frac{1}{R_{1}} & 0 \\
-\frac{1}{R} & -\frac{1}{R_{1}} & \frac{1}{R}+\frac{1}{R_{1}}+0+\frac{1}{R_{2}} & -\frac{1}{R_{2}} \\
0 & 0 & 0-\frac{A_{v 2}}{R_{o 2}} & 0+\frac{1}{R_{o 2}}
\end{array}\right]\left[\begin{array}{c}
v_{k 1} \\
v_{k 2} \\
v_{k 3} \\
v_{o}
\end{array}\right]=\left[\begin{array}{c}
\frac{v_{s 1}}{R_{i 1}} \\
\frac{A_{v 1} v_{s 1}}{R_{o 1}} \\
\frac{v_{s 2}}{R_{i 2}} \\
\frac{A_{v 2} v_{s 2}}{R_{o 2}}
\end{array}\right] .
$$

(Step 6) Multiplying rows 2 and 4 of Eq. (4) by $R_{o 1} / A_{v 1}$ and $R_{o 2} / A_{v 2}$, respectively, and applying L'Hospital's rule give the following simplified result:

$$
\left[\begin{array}{cccc}
\frac{1}{R_{2}}+\frac{1}{R_{1}}+\frac{1}{R} & -\frac{1}{R_{1}} & -\frac{1}{R} & 0 \\
-1 & 0 & 0 & 0 \\
-\frac{1}{R} & -\frac{1}{R_{1}} & \frac{1}{R}+\frac{1}{R_{1}}+\frac{1}{R_{2}} & -\frac{1}{R_{2}} \\
0 & 0 & -1 & 0
\end{array}\right]\left[\begin{array}{c}
v_{k 1} \\
v_{k 2} \\
v_{k 3} \\
v_{o}
\end{array}\right]=\left[\begin{array}{c}
0 \\
-v_{s 1} \\
0 \\
-v_{s 2}
\end{array}\right]
$$

(Remark 2) A significant feature of this study is the use of L'Hospital's rule and the movingterm techniques to solve complicated operational amplifier systems with a dependent source, ${ }^{(18)}$ where 


$$
\begin{gathered}
-\frac{A_{v 1} v_{s 1}}{R_{o 1}} \times\left(\frac{R_{o 1}}{A_{v 1}}\right)=-v_{s 1}, \frac{1}{R_{o 1}} \times\left(\frac{R_{o 1}}{A_{v 1}}\right)=0,-\frac{1}{R_{1}} \times\left(\frac{R_{o 1}}{A_{v 1}}\right)=0, \\
-\frac{A_{v 2} v_{s 2}}{R_{o 2}} \times\left(\frac{R_{o 2}}{A_{v 2}}\right)=-v_{s 2}, \frac{1}{R_{o 2}} \times\left(\frac{R_{o 2}}{A_{v 2}}\right)=0 .
\end{gathered}
$$

(Step 7) Utilizing our previously invented simple matrix operations simplifies the derivation process. ${ }^{(3)}$ Multiplying rows 1 and 2 of Eq. (5) by $-R_{1}$ and $-v_{s 2} / v_{v 1}$, respectively, give the following simplified result:

$$
\left[\begin{array}{cccc}
\frac{1}{R_{2}}+\frac{1}{R_{1}}+\frac{1}{R} & -\frac{1}{R_{1}} & -\frac{1}{R} & 0 \\
-1 & 0 & 0 & 0 \\
-\frac{2}{R}-\frac{1}{R_{1}}-\frac{1}{R_{2}} & 0 & \frac{2}{R}+\frac{1}{R_{1}}+\frac{1}{R_{2}} & -\frac{1}{R_{2}} \\
\frac{v_{s 2}}{v_{s 1}} & 0 & -1 & 0
\end{array}\right]\left[\begin{array}{c}
v_{k 1} \\
v_{k 2} \\
v_{k 3} \\
v_{o}
\end{array}\right]=\left[\begin{array}{c}
0 \\
-v_{s 1} \\
0 \\
0
\end{array}\right]
$$

(Remark 3) To reduce the difficulty in solving complicated operational amplifier systems, we newly propose the above useful matrix operations for use in existing approaches. ${ }^{(15,21)}$

(Step 8) Apply Cramer's rule to obtain the following desired function:

$$
v_{o}=R_{2}\left(\frac{1}{R_{2}}+\frac{1}{R_{1}}+\frac{2}{R}\right)\left(v_{s 2}-v_{s 1}\right)=\left(v_{s 2}-v_{s 1}\right)[4 k+3] .
$$

(Step 9) To find the Thevenin equivalent circuit between nodes $v_{k 1}$ and $v_{k 2}$, place a current (voltage) source $i_{t}\left(v_{t}\right)$ of the desired terminal pair as shown in Fig. 3.

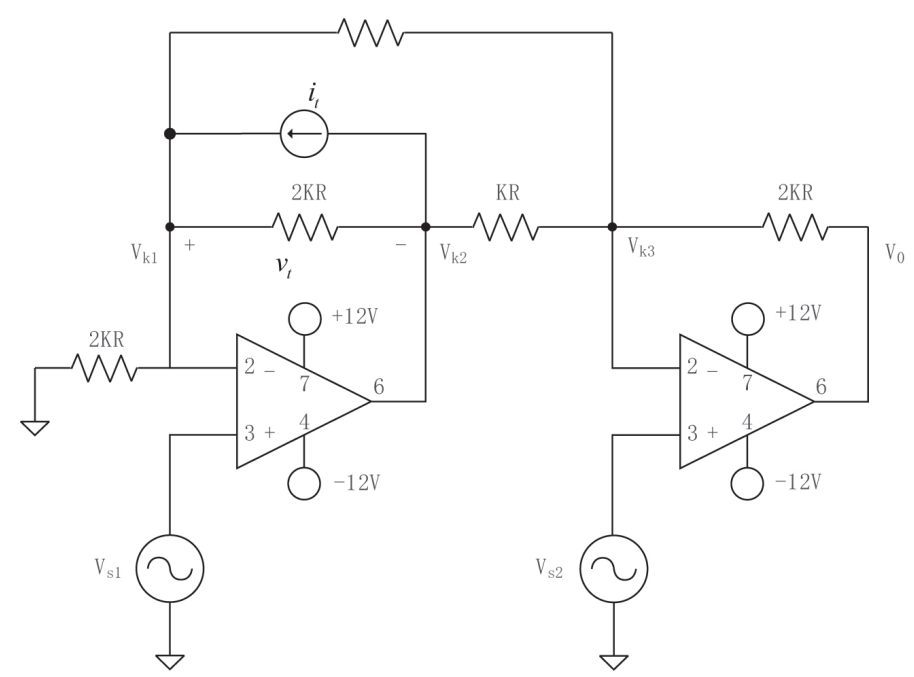

Fig. 3. Demonstrated example of finding the Thevenin equivalent circuit. 
Apply the above processes to derive the variables $v_{t}\left(i_{t}\right)$ and then rearrange the variables $v_{t}\left(i_{t}\right)$ in the form

$$
v_{t}=i_{t}\left(R_{T H}\right)+V_{T H} .
$$

Hence, we obtain two Thevenin equivalent parameters simultaneously. The node-voltage matrix equations corresponding to Fig. 3 are given as

$$
\left[\begin{array}{cccc}
\frac{1}{R_{2}}+\frac{1}{R_{i 1}}+\frac{1}{R_{1}}+\frac{1}{R} & -\frac{1}{R_{1}} & -\frac{1}{R} & 0 \\
-\frac{1}{R_{1}} & \frac{2}{R_{1}}+\frac{1}{R_{o 1}} & -\frac{1}{R_{1}} & 0 \\
-\frac{1}{R} & -\frac{1}{R_{1}} & \frac{1}{R}+\frac{1}{R_{1}}+\frac{1}{R_{i 2}}+\frac{1}{R_{2}} & -\frac{1}{R_{2}} \\
0 & 0 & -\frac{1}{R_{2}} & \frac{1}{R_{2}}+\frac{1}{R_{o 2}}
\end{array}\right]\left[\begin{array}{c}
v_{k 1} \\
v_{k 2} \\
v_{k 3} \\
v_{o}
\end{array}\right]=\left[\begin{array}{c}
\frac{v_{s 1}}{R_{i 1}}+i_{t} \\
\frac{A_{v 1} v_{i 1}}{R_{o 1}}-i_{t} \\
\frac{v_{s 2}}{R_{i 2}} \\
\frac{A_{v 2} v_{i 2}}{R_{o 2}}
\end{array}\right]
$$

Substituting $v_{i 1}=v_{k 1}-v_{s 1}$ and $v_{i 2}=v_{k 3}-v_{s 2}$ into Eq. (9) and moving the node-voltage variables to the other side of the equation yield the following expression:

$$
\left[\begin{array}{cccc}
\frac{1}{R_{2}}+\frac{1}{R_{i 1}}+\frac{1}{R_{1}}+\frac{1}{R} & -\frac{1}{R_{1}} & -\frac{1}{R} & 0 \\
-\frac{1}{R_{1}}-\frac{A_{v 1}}{R_{o 1}} & \frac{2}{R_{1}}+\frac{1}{R_{o 1}} & -\frac{1}{R_{1}} & 0 \\
-\frac{1}{R} & -\frac{1}{R_{1}} & \frac{1}{R}+\frac{1}{R_{1}}+\frac{1}{R_{i 2}}+\frac{1}{R_{2}} & -\frac{1}{R_{2}} \\
0 & 0 & -\frac{1}{R_{2}}-\frac{A_{v 2}}{R_{o 2}} & \frac{1}{R_{2}}+\frac{1}{R_{o 2}}
\end{array}\right]\left[\begin{array}{l}
v_{k 1} \\
v_{k 2} \\
v_{k 3} \\
v_{o}
\end{array}\right]=\left[\begin{array}{c}
\frac{v_{s 1}}{R_{i 1}}+i_{t} \\
-\frac{A_{v 1} v_{s 1}}{R_{o 1}}-i_{t} \\
\frac{v_{s 2}}{R_{i 2}} \\
-\frac{A_{v 2} v_{s 2}}{R_{o 2}}
\end{array}\right]
$$

Multiplying rows 2 and 4 of Eq. (10) by $R_{o 1} / A_{v 1}$ and $R_{o 2} / A_{v 2}$, respectively, and applying L'Hospital's rule give the following simplified result:

$$
\left[\begin{array}{cccc}
\frac{1}{R_{2}}+\frac{1}{R_{1}}+\frac{1}{R} & -\frac{1}{R_{1}} & -\frac{1}{R} & 0 \\
-1 & 0 & 0 & 0 \\
-\frac{1}{R} & -\frac{1}{R_{1}} & \frac{1}{R}+\frac{1}{R_{1}}+\frac{1}{R_{2}} & -\frac{1}{R_{2}} \\
0 & 0 & -1 & 0
\end{array}\right]\left[\begin{array}{c}
v_{k 1} \\
v_{k 2} \\
v_{k 3} \\
v_{o}
\end{array}\right]=\left[\begin{array}{c}
i_{t} \\
-v_{s 1} \\
0 \\
-v_{s 2}
\end{array}\right]
$$


Applying our previously invented reduced-order operations simplifies the derivation process. ${ }^{(3)}$

$$
\left[\begin{array}{ccc}
\frac{1}{R_{2}}+\frac{1}{R_{1}}+\frac{1}{R} & -\frac{1}{R_{1}} & -\frac{1}{R} \\
-1 & 0 & 0 \\
0 & 0 & -1
\end{array}\right]\left[\begin{array}{c}
v_{k 1} \\
v_{k 2} \\
v_{k 3}
\end{array}\right]=\left[\begin{array}{c}
i_{t} \\
-v_{s 1} \\
-v_{s 2}
\end{array}\right]
$$

Utilizing our previously invented column operations simplifies the derivation process. ${ }^{(3)}$ Adding column 1 to column 2 of the above equation gives the following simplified result:

$$
\left[\begin{array}{ccc}
\frac{1}{R_{2}}+\frac{1}{R_{1}}+\frac{1}{R} & \frac{1}{R_{2}}+\frac{1}{R} & -\frac{1}{R} \\
-1 & -1 & 0 \\
0 & 0 & -1
\end{array}\right]\left[\begin{array}{c}
v_{k 1}-v_{k 2} \\
v_{k 2} \\
v_{k 3}
\end{array}\right]=\left[\begin{array}{c}
i_{t} \\
-v_{s 1} \\
-v_{s 2}
\end{array}\right]
$$

Multiply row 1 of the above equation by $-R$. Adding row 1 to row 3 gives the following simplified result:

$$
\left[\begin{array}{ccc}
\frac{1}{R_{2}}+\frac{1}{R_{1}}+\frac{1}{R} & \frac{1}{R_{2}}+\frac{1}{R} & -\frac{1}{R} \\
-1 & -1 & 0 \\
-\frac{R}{R_{2}}-\frac{R}{R_{1}}-1 & -\frac{R}{R_{2}}-1 & 0
\end{array}\right]\left[\begin{array}{c}
v_{k 1}-v_{k 2} \\
v_{k 2} \\
v_{k 3}
\end{array}\right]=\left[\begin{array}{c}
i_{t} \\
-v_{s 1} \\
-v_{s 2}-i_{t} R
\end{array}\right] .
$$

Applying our previously invented reduced-order operations simplifies the derivation process. $^{(3)}$

$$
\left[\begin{array}{cc}
-1 & -1 \\
-\frac{R}{R_{2}}-\frac{R}{R_{1}}-1 & -\frac{R}{R_{2}}-1
\end{array}\right]\left[\begin{array}{c}
v_{k 1}-v_{k 2}\left(i . e . v_{t}\right) \\
v_{k 2}
\end{array}\right]=\left[\begin{array}{c}
-v_{s 1} \\
-v_{s 2}-R i_{t}
\end{array}\right]
$$

Cramer's rule is applied to obtain the desired function

$$
v_{t}=i_{t}\left(-R_{1}\right)+\left(-\frac{R_{1}}{R} v_{s 2}+\left(\frac{R_{1}}{R_{2}}+\frac{R_{1}}{R} v_{s 1}\right)\right) \text {, }
$$

i.e.,

$$
V_{T H}=-\frac{R_{1}}{R} v_{s 2}+\left(\frac{R_{1}}{R_{2}}+\frac{R_{1}}{R} v_{s 1}\right), R_{T H}=-R_{1} .
$$


It is also worth mentioning that, so far, we have never seen a systematically solved matrix solution for operational amplifier circuits to our best knowledge. To exploit the fact that the proposed complicated operational amplifier approach is more useful than the traditional approach of using the disorganized virtual ground technique and Kirchhoff's voltage law (Kirchhoff's current law), the traditional approach is outlined as follows.

We redrew Fig. 1 as Fig. 4 with all node voltages and branch currents. Applying the virtual ground technique and Ohm's law yields

$$
I=\frac{v_{S 1}}{2 K R}
$$

and

$$
I_{1}=\frac{v_{s 2}-v_{s 1}}{R}
$$

From Kirchhoff's current and Kirchhoff's voltage laws, we obtain

$$
I_{2}=I-I_{1}=\frac{v_{s 1}}{2 K R}-\frac{v_{s 2}-v_{s 1}}{R}=\frac{v_{s 1}(2 K+1)-2 K v_{s 2}}{2 K R}
$$

and

$$
\begin{aligned}
& V^{\prime}=v_{s 1}+I_{2} K R=v_{s 1}+\frac{v_{s 1}(2 K+1)-2 K v_{s 2}}{2 K R}(K R) \\
& =v_{s 1}(K+(3 / 2))+v_{s 2}(-K) .
\end{aligned}
$$

Ohm's law is used to obtain

$$
\begin{aligned}
& I_{3}=\frac{v_{s 2}-V^{\prime}}{K R}=\frac{v_{s 2}-\left[v_{s 1}(K+(3 / 2))+v_{s 2}(-K)\right]}{K R} \\
& =v_{s 1}\left(-\frac{1}{R}-\frac{3}{2 K R}\right)+v_{s 2}\left(\frac{1}{R}+\frac{1}{K R}\right) .
\end{aligned}
$$

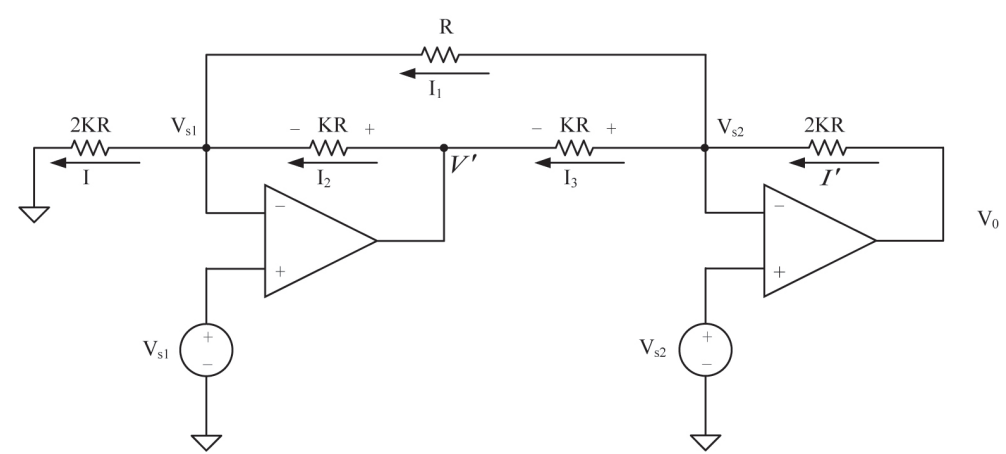

Fig. 4. Demonstrated example of a complicated operational amplifier system. 
Thus, we obtain the following equation via Kirchhoff's current law:

$$
\begin{aligned}
I^{\prime} & =I_{1}+I_{3}=\frac{v_{s 2}-v_{s 1}}{R}+\left[v_{s 1}\left(-\frac{1}{R}-\frac{3}{2 K R}\right)+v_{s 2}\left(\frac{1}{R}+\frac{1}{K R}\right)\right] \\
& =v_{s 1}\left(-\frac{2}{R}-\frac{3}{2 K R}\right)+v_{s 2}\left(\frac{2}{R}+\frac{1}{K R}\right) .
\end{aligned}
$$

Finally, applying Kirchhoff’s voltage law gives

$$
\begin{aligned}
V_{o} & =I^{\prime}(2 K R)+v_{s 2}=\left[v_{s 1}\left(-\frac{2}{R}-\frac{3}{2 K R}\right)+v_{s 2}\left(\frac{2}{R}+\frac{1}{K R}\right)\right]+v_{s 2} \\
& =(4 K+3)\left(v_{s 2}-v_{s 1}\right) .
\end{aligned}
$$

\section{Comparative Example}

To show the significant merits of our method over traditional approaches, a comparative example is given in this section.

Consider the complicated operational amplifier system shown in Fig. 5. Firstly, applying the traditional approach of using the disorganized virtual ground technique and Kirchhoff's voltage law (Kirchhoff's current law) solves the above system. Utilizing the virtual ground technique with $I=0$ gives

$$
I_{1}=I_{2}=\frac{V_{s}}{K R}
$$

The virtual ground technique with $V_{+}=V_{-}=0$ is applied to obtain

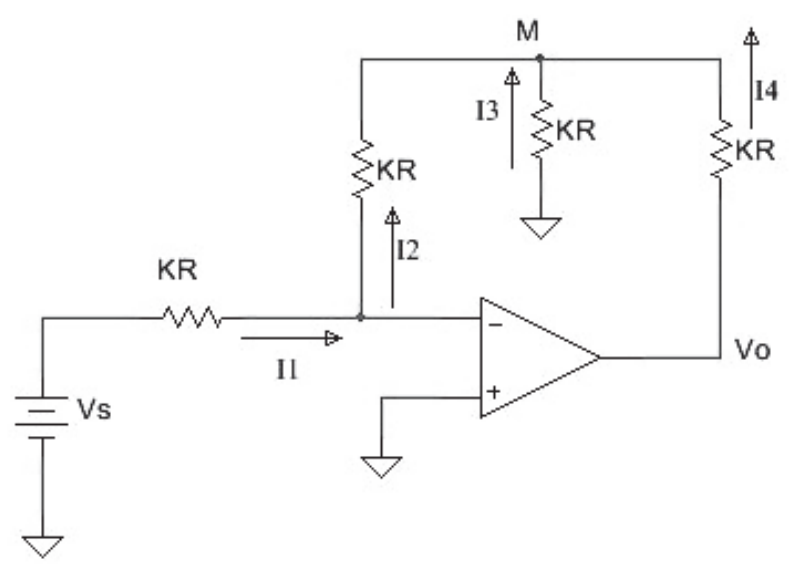

Fig. 5. Complicated operational amplifier system used for comparison. 


$$
I_{1}=I_{2}=\frac{V_{s}}{K R}
$$

and

$$
V_{M}=-I_{2}(K R)
$$

Equations (24)-(26) are combined to give

$$
V_{M}=V_{-}-I_{2}(K R)=-V_{s}
$$

and

$$
I_{3}=\frac{0-V_{M}}{K R}=\frac{V_{s}}{K R} .
$$

Kirchhoff's voltage and Kirchhoff's current laws are respectively used to obtain

$$
V_{o}=I_{4} K R-I_{2} K R
$$

and

$$
I_{2}+I_{3}=-I_{4}
$$

Thus,

$$
V_{o}=I_{4} K R-I_{2} K R=\left(-I_{2}-I_{3}\right) K R-I_{2} K R=-I_{3} K R-2 I_{2} K R .
$$

Substituting Eqs. (25) and (28) into Eq. (31) gives

$$
V_{o}=-I_{3} K R-2 I_{2} K R=-\left(\frac{V_{s}}{K R}\right) K R-2\left(\frac{V_{s}}{K R}\right) K R=-3 V_{s} .
$$

Thus,

$$
\frac{V_{o}}{V_{s}}=-3
$$

As a comparison with the above traditional approach, we use the proposed complicated operational amplifier approach to easily solve the above comparative example in only three steps as follows. Firstly, an equivalent circuit of Fig. 5 is drawn in Fig. 6. 


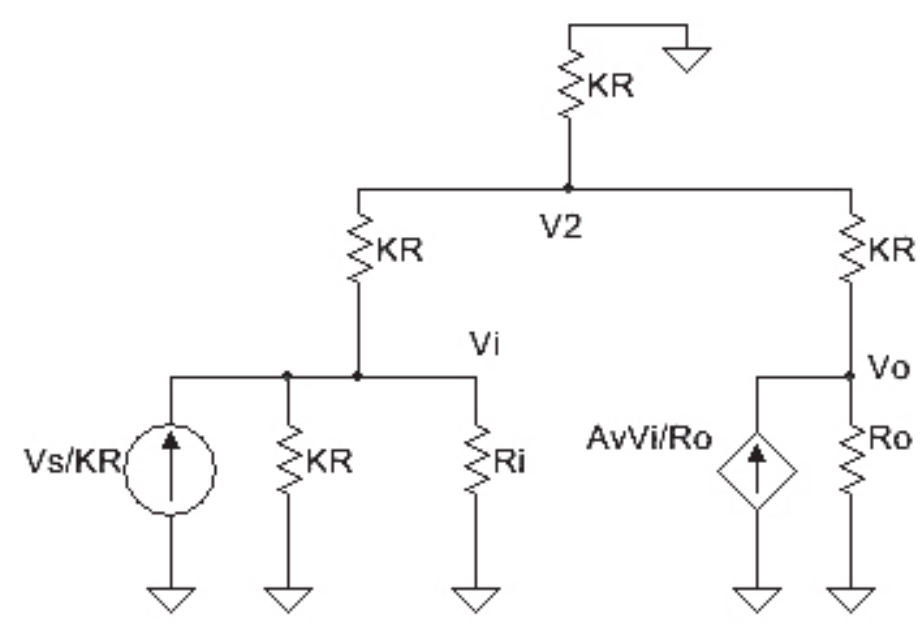

Fig. 6. Equivalent circuit of Fig. 5.

The node voltage equations are directly listed as follows via the proposed complicated operational amplifier approach:

$$
\left[\begin{array}{ccc}
\frac{2}{k R}+\frac{1}{R_{i}} & -\frac{1}{k R} & 0 \\
-\frac{1}{k R} & \frac{3}{k R} & -\frac{1}{k R} \\
0 & -\frac{1}{k R} & \frac{1}{k R}+\frac{1}{R_{o}}
\end{array}\right]\left[\begin{array}{c}
v_{1} \\
v_{2} \\
v_{o}
\end{array}\right]=\left[\begin{array}{c}
\frac{v_{s}}{k R} \\
0 \\
\frac{A_{v} v_{i}}{R_{o}}
\end{array}\right]
$$

Multiplying the third row by $R_{o} / A_{v}$ and applying the L'Hospital rule give

$$
\left[\begin{array}{ccc}
\frac{2}{k R} & -\frac{1}{k R} & 0 \\
-\frac{1}{k R} & \frac{3}{k R} & -\frac{1}{k R} \\
-1 & 0 & 0
\end{array}\right]\left[\begin{array}{c}
v_{1} \\
v_{2} \\
v_{o}
\end{array}\right]=\left[\begin{array}{c}
\frac{v_{s}}{k R} \\
0 \\
0
\end{array}\right]
$$

Finally, Cramer's rule is applied to obtain the desired function

$$
\frac{V_{o}}{V_{s}}=-3
$$

(Remark 4) From the above comparative example, it is clear that the use of the traditional approach is very confusing. In particular, for more complicated operational amplifier systems with two dependent elements such as the demonstrated example (Fig. 1), it is 
difficult to solve the desired function. In contrast, the use of the proposed complicated operational amplifier approach is very simple and efficient. A system can even be solved in only three steps.

\section{Implementation of Proposed Approach of an Online ECG Device}

To demonstrate the practical applicability of our proposed approach, we investigate its implementation in an online ECG system.

\subsection{AD620 instrumentation amplifier}

Consider the well-known instrumentation amplifier with the operational amplifier shown in Fig. 7. ${ }^{(2)}$ The AD620 instrumentation amplifier is a low-cost and high-accuracy device that requires only one external resistor to adjust voltage gains. Furthermore, the characteristics of the AD620 instrumentation amplifier including the low input bias current, low disturbance, and low power make it well suited for biomedical devices such as ECG and blood pressure monitors. We will separate the input part from the output part in the analysis in the following derivations. The procedures of the proposed effective approach are used to obtain the desired performance as follows, where Figs. 8 and 9 are the equivalent circuits for the input and output parts of the AD620 instrumentation amplifier, respectively.

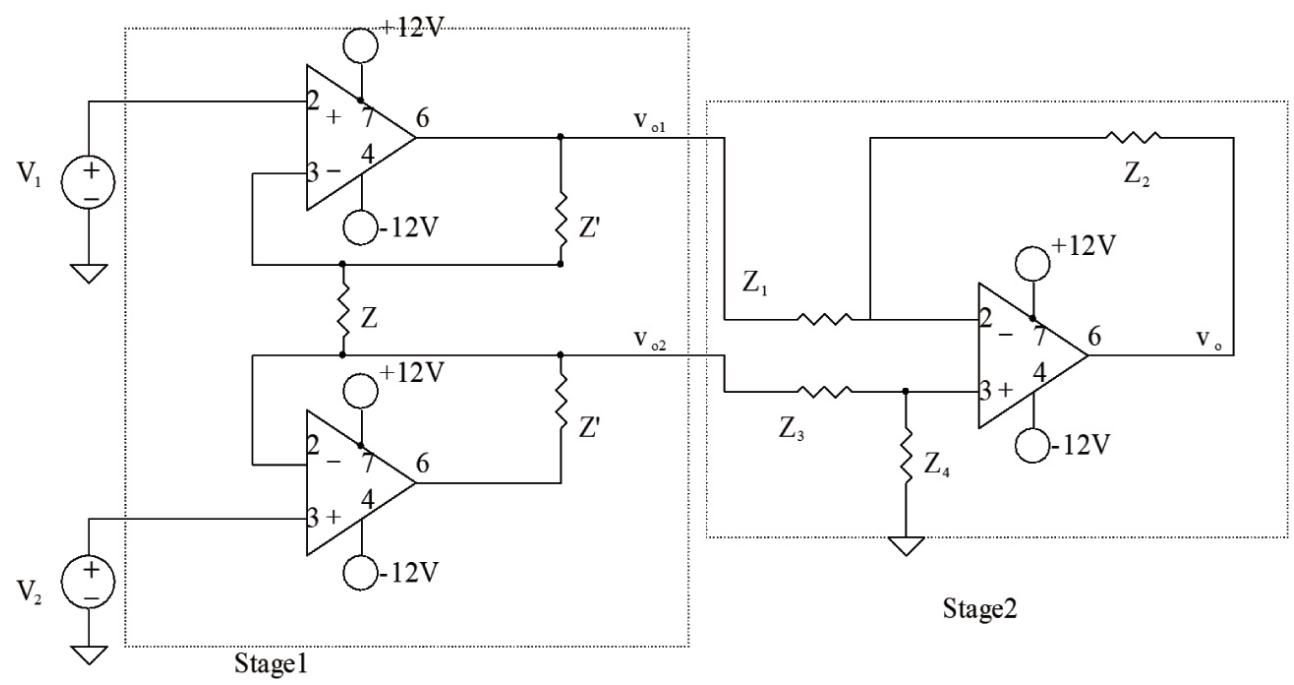

Fig. 7. AD620 instrumentation amplifier. 
(Input part of AD620 instrumentation amplifier)

(Step 1)

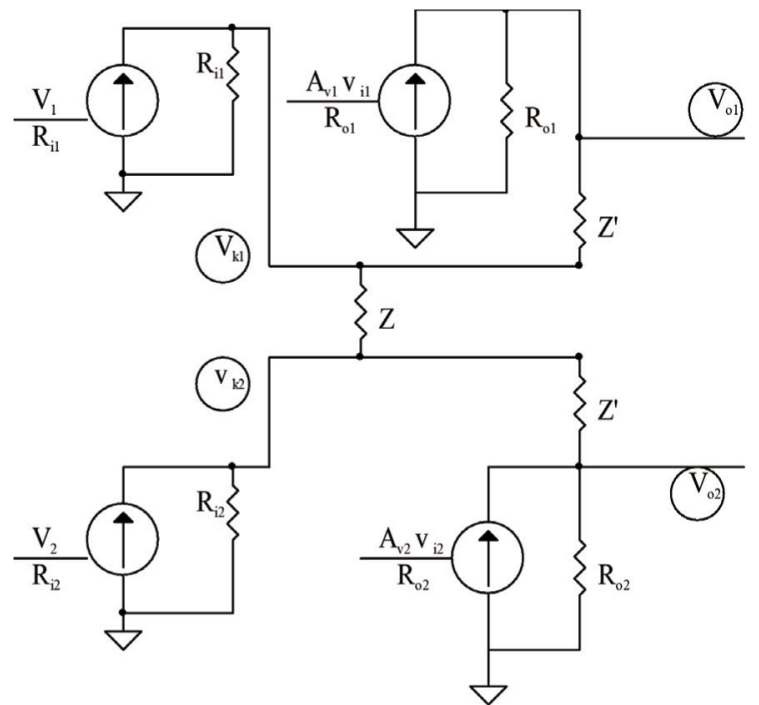

Fig. 8. Equivalent circuit for input part of AD620 instrumentation amplifier.

(Step 2)

$$
\left[\begin{array}{cccc}
\frac{1}{R_{i 1}}+\frac{1}{Z^{\prime}}+\frac{1}{Z} & -\frac{1}{Z} & -\frac{1}{Z^{\prime}} & 0 \\
-\frac{1}{Z} & \frac{1}{R_{i 2}}+\frac{1}{Z^{\prime}}+\frac{1}{Z} & 0 & -\frac{1}{Z^{\prime}} \\
-\frac{1}{Z^{\prime}} & 0 & \frac{1}{R_{o 1}}+\frac{1}{Z^{\prime}} & 0 \\
0 & -\frac{1}{Z^{\prime}} & 0 & \frac{1}{R_{o 2}}+\frac{1}{Z^{\prime}}
\end{array}\right]\left[\begin{array}{c}
V_{k 1} \\
V_{k 2} \\
V_{o 1} \\
V_{o 2}
\end{array}\right]=\left[\begin{array}{c}
\frac{V_{1}}{R_{i 1}} \\
\frac{V_{2}}{R_{i 2}} \\
\frac{A_{V 1} V_{i 1}}{R_{o 1}} \\
\frac{A_{V 2} V_{i 2}}{R_{o 2}}
\end{array}\right]
$$

(Step 3) and (Step 4)

$$
\left[\begin{array}{cccc}
\frac{1}{R_{i 1}}+\frac{1}{Z^{\prime}}+\frac{1}{Z} & -\frac{1}{Z} & -\frac{1}{Z^{\prime}} & 0 \\
-\frac{1}{Z} & \frac{1}{R_{i 2}}+\frac{1}{Z^{\prime}}+\frac{1}{Z} & 0 & -\frac{1}{Z^{\prime}} \\
-\frac{1}{Z^{\prime}}-\frac{A_{V 1}}{R_{o 1}} & 0 & \frac{1}{R_{o 1}}+\frac{1}{Z^{\prime}} & 0 \\
0 & -\frac{1}{Z^{\prime}}-\frac{A_{V 2}}{R_{o 2}} & 0 & \frac{1}{R_{o 2}}+\frac{1}{Z^{\prime}}
\end{array}\right]\left[\begin{array}{c}
V_{k 1} \\
V_{k 2} \\
V_{o 1} \\
V_{o 2}
\end{array}\right]=\left[\begin{array}{c}
\frac{V_{1}}{R_{i 1}} \\
\frac{V_{2}}{R_{i 2}} \\
-\frac{A_{V 1} V_{1}}{R_{o 1}} \\
-\frac{A_{V 2} V_{2}}{R_{o 2}}
\end{array}\right]
$$


(Step 5)

$$
\left[\begin{array}{cccc}
0+\frac{1}{Z^{\prime}}+\frac{1}{Z} & -\frac{1}{Z} & -\frac{1}{Z^{\prime}} & 0 \\
-\frac{1}{Z} & 0+\frac{1}{Z^{\prime}}+\frac{1}{Z} & 0 & -\frac{1}{Z^{\prime}} \\
-\frac{A_{V 1}}{R_{o 1}} & 0 & \frac{1}{R_{o 1}} & 0 \\
0 & -\frac{A_{V 2}}{R_{o 2}} & 0 & \frac{1}{R_{o 2}}
\end{array}\right]\left[\begin{array}{c}
V_{k 1} \\
V_{k 2} \\
V_{o 1} \\
V_{o 2}
\end{array}\right]=\left[\begin{array}{c}
0 \\
0 \\
-\frac{A_{V 1} V_{1}}{R_{o 1}} \\
-\frac{A_{V 2} V_{2}}{R_{o 2}}
\end{array}\right]
$$

(Step 6)

$$
\left[\begin{array}{cccc}
Z+Z^{\prime} & -Z^{\prime} & -Z & 0 \\
-Z^{\prime} & Z+Z^{\prime} & 0 & -Z \\
-1 & 0 & 0 & 0 \\
0 & -1 & 0 & 0
\end{array}\right]\left[\begin{array}{c}
V_{k 1} \\
V_{k 2} \\
V_{o 1} \\
V_{o 2}
\end{array}\right]=\left[\begin{array}{c}
0 \\
0 \\
-V_{1} \\
-V_{2}
\end{array}\right]
$$

(Step 7)

$$
\left[\begin{array}{cccc}
Z+Z^{\prime} & -Z^{\prime} & -Z & 0 \\
-Z^{\prime} & Z+Z^{\prime} & 0 & -Z \\
-1 & 0 & 0 & 0 \\
\frac{V_{2}}{V_{1}} & -1 & 0 & 0
\end{array}\right]\left[\begin{array}{c}
V_{k 1} \\
V_{k 2} \\
V_{o 1} \\
V_{o 2}
\end{array}\right]=\left[\begin{array}{c}
0 \\
0 \\
-V_{1} \\
0
\end{array}\right]
$$

(Step 8)

$$
V_{o 1}=V_{1}\left(\frac{Z+Z}{Z}\right)+V_{2}\left(-\frac{Z}{Z}\right)
$$

and

$$
V_{o 2}=V_{1}\left(-\frac{Z}{Z}\right)+V_{2}\left(\frac{Z+Z}{Z}\right)
$$


(Output part of AD620 instrumentation amplifier)

(Step 1)

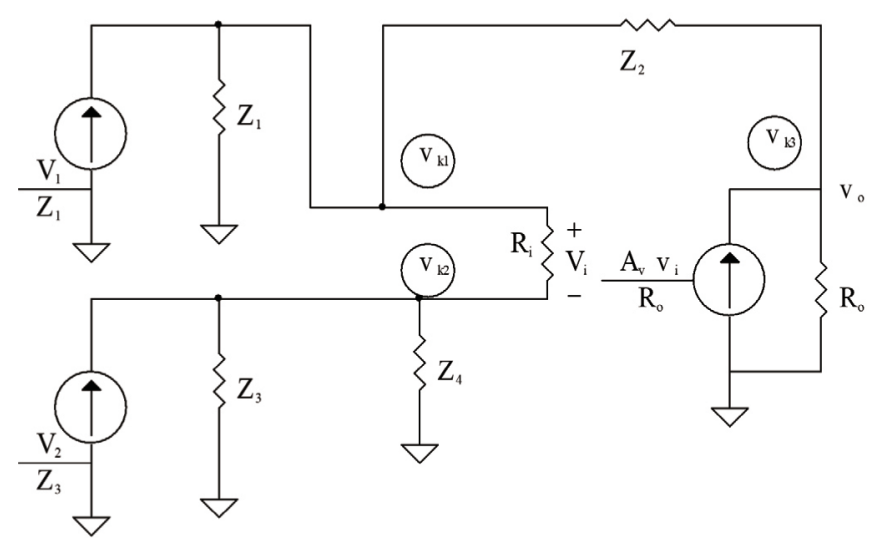

Fig. 9. Equivalent circuit for output part of AD620 instrumentation amplifier.

(Step 2)

$$
\left[\begin{array}{ccc}
\frac{1}{Z_{1}}+\frac{1}{R_{i}}+\frac{1}{Z_{2}} & -\frac{1}{R_{i}} & -\frac{1}{Z_{2}} \\
-\frac{1}{R_{i}} & \frac{1}{Z_{3}}+\frac{1}{R_{i}}+\frac{1}{Z_{4}} & 0 \\
-\frac{1}{Z_{2}} & 0 & \frac{1}{Z_{2}}+\frac{1}{R_{o}}
\end{array}\right]\left[\begin{array}{c}
V_{k 1} \\
V_{k 2} \\
V_{o}
\end{array}\right]=\left[\begin{array}{c}
\frac{V_{1}}{Z_{1}} \\
\frac{V_{2}}{Z_{3}} \\
\frac{A_{v} V_{i}}{R_{o}}
\end{array}\right]
$$

(Step 3) and (Step 4)

$$
\left[\begin{array}{ccc}
\frac{1}{Z_{1}}+\frac{1}{R_{i}}+\frac{1}{Z_{2}} & -\frac{1}{R_{i}} & -\frac{1}{Z_{2}} \\
-\frac{1}{R_{i}} & \frac{1}{Z_{3}}+\frac{1}{R_{i}}+\frac{1}{Z_{4}} & 0 \\
-\frac{1}{Z_{2}}-\frac{A_{v}}{R_{o}} & \frac{A_{v}}{R_{o}} & \frac{1}{Z_{2}}+\frac{1}{R_{o}}
\end{array}\right]\left[\begin{array}{c}
V_{k 1} \\
V_{k 2} \\
V_{o}
\end{array}\right]=\left[\begin{array}{c}
\frac{V_{1}}{Z_{1}} \\
\frac{V_{2}}{Z_{3}} \\
0
\end{array}\right]
$$

(Step 5)

$$
\left[\begin{array}{ccc}
\frac{1}{Z_{1}}+0+\frac{1}{Z_{2}} & 0 & -\frac{1}{Z_{2}} \\
0 & \frac{1}{Z_{3}}+0+\frac{1}{Z_{4}} & 0 \\
-\frac{1}{Z_{2}}-\frac{A_{v}}{R_{o}} & \frac{A_{v}}{R_{o}} & \frac{1}{Z_{2}}+\frac{1}{R_{o}}
\end{array}\right]\left[\begin{array}{c}
V_{k 1} \\
V_{k 2} \\
V_{o}
\end{array}\right]=\left[\begin{array}{c}
\frac{V_{1}}{Z_{1}} \\
\frac{V_{2}}{Z_{3}} \\
0
\end{array}\right]
$$


(Step 6)

$$
\left[\begin{array}{ccc}
\frac{1}{Z_{1}}+\frac{1}{Z_{2}} & 0 & -\frac{1}{Z_{2}} \\
0 & \frac{1}{Z_{3}}+\frac{1}{Z_{4}} & 0 \\
-1 & 1 & 0
\end{array}\right]\left[\begin{array}{c}
V_{k 1} \\
V_{k 2} \\
V_{o}
\end{array}\right]=\left[\begin{array}{c}
\frac{V_{1}}{Z_{1}} \\
\frac{V_{2}}{Z_{3}} \\
0
\end{array}\right]
$$

(Step 7)

$$
\left[\begin{array}{ccc}
\frac{1}{Z_{1}}+\frac{1}{Z_{2}} & 0 & -\frac{1}{Z_{2}} \\
\frac{1}{Z_{3}}+\frac{1}{Z_{4}} & 0 & 0 \\
-1 & 1 & 0
\end{array}\right]\left[\begin{array}{l}
V_{k 1} \\
V_{k 2} \\
V_{o}
\end{array}\right]=\left[\begin{array}{c}
\frac{V_{1}}{Z_{1}} \\
\frac{V_{2}}{Z_{3}} \\
0
\end{array}\right]
$$

(Step 8)

$$
V_{o}=\left(-\frac{Z_{2}}{Z_{1}}\right) V_{1}+\left(\frac{1+\frac{Z_{2}}{Z_{1}}}{1+\frac{Z_{3}}{Z_{4}}}\right) V_{2}
$$

\section{2 $60 \mathrm{~Hz}$ notch filter}

After the analog ECG signal is measured and transferred into the digital mode, the notch filter can be used to reduce the power of the $60 \mathrm{~Hz}$ embedded noise. ${ }^{(9)}$ Consider the wellknown $60 \mathrm{~Hz}$ notch filter with the operator amplifier shown in Fig. 10, where $R_{1}=R_{2}=10 \mathrm{k} \Omega$,

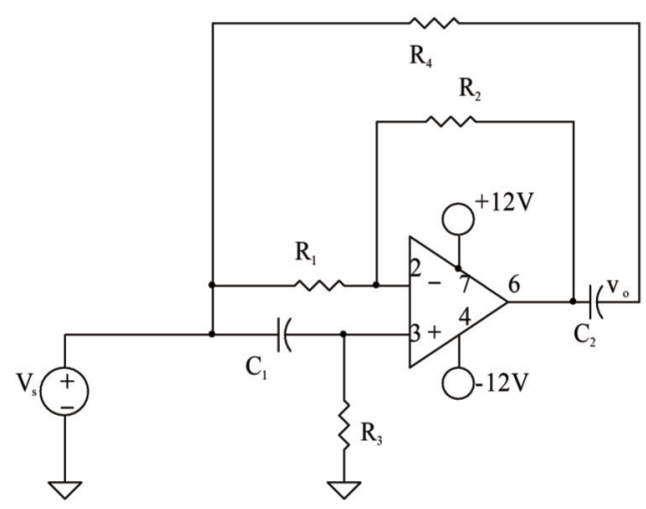

Fig. 10. $60 \mathrm{~Hz}$ notch filter. 
$R_{3}=R_{4} \equiv R=22 \mathrm{k} \Omega$, and $C_{1}=C_{2} \equiv C=110 \mathrm{nF}$. The procedures of the proposed effective approach are applied to obtain the notch filter property as follows, where Fig. 11 is the equivalent circuit of the $60 \mathrm{~Hz}$ notch filter in Fig. 10.

(Step 1)

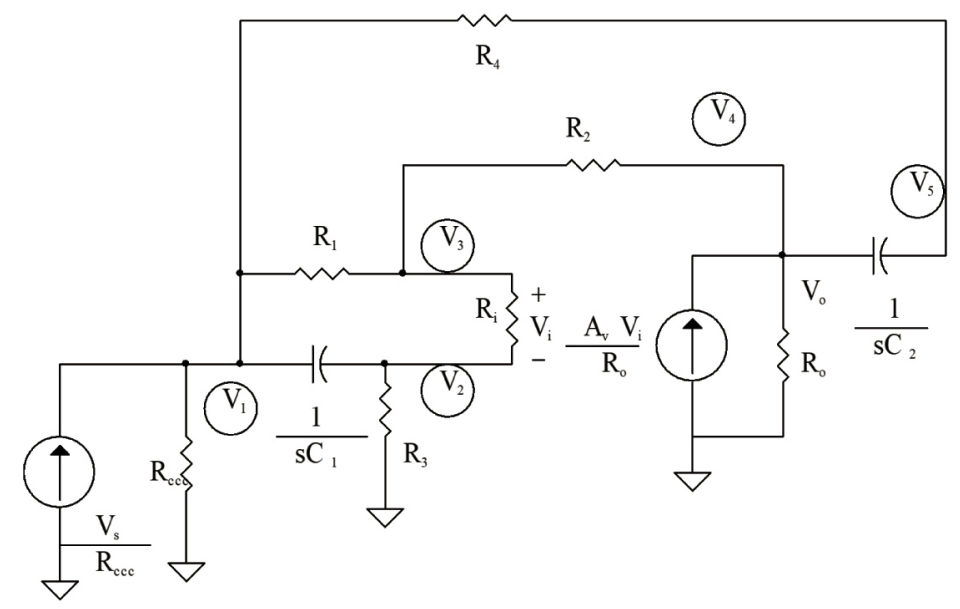

Fig. 11. Equivalent circuit of the $60 \mathrm{~Hz}$ notch filter in Fig. 10.

(Step 2)

$$
\left[\begin{array}{ccccc}
\frac{1}{R_{c c c}}+\frac{1}{R_{1}}+s C_{1}+\frac{1}{R_{4}} & -s C_{1} & -\frac{1}{R_{1}} & 0 & -\frac{1}{R_{4}} \\
-s C_{1} & \frac{1}{R_{i}}+\frac{1}{R_{3}}+s C_{1} & -\frac{1}{R_{i}} & 0 & 0 \\
-\frac{1}{R_{1}} & -\frac{1}{R_{i}} & \frac{1}{R_{i}}+\frac{1}{R_{1}}+\frac{1}{R_{2}} & -\frac{1}{R_{2}} & 0 \\
0 & 0 & -\frac{1}{R_{2}} & \frac{1}{R_{o}}+\frac{1}{R_{2}}+s C_{2} & -s C_{2} \\
-\frac{1}{R_{4}} & 0 & 0 & -s C_{2} & \frac{1}{R_{4}}+s C_{2}
\end{array}\right]\left[\begin{array}{c}
V_{1} \\
V_{2} \\
V_{3} \\
V_{4} \\
V_{5}
\end{array}\right]=\left[\begin{array}{c}
\frac{V_{s}}{R_{c c c}} \\
0 \\
0 \\
\frac{A_{v} V_{i}}{R_{o}} \\
0
\end{array}\right]
$$

(Step 3)

$$
\left[\begin{array}{ccccc}
1 & 0 & 0 & 0 & 0 \\
-s C_{1} & \frac{1}{R_{i}}+\frac{1}{R_{3}}+s C_{1} & -\frac{1}{R_{i}} & 0 & 0 \\
-\frac{1}{R_{1}} & -\frac{1}{R_{i}} & \frac{1}{R_{i}}+\frac{1}{R_{1}}+\frac{1}{R_{2}} & -\frac{1}{R_{2}} & 0 \\
0 & 0 & -\frac{1}{R_{2}} & \frac{1}{R_{o}}+\frac{1}{R_{2}}+s C_{2} & -s C_{2} \\
-\frac{1}{R_{4}} & 0 & 0 & -s C_{2} & \frac{1}{R_{4}}+s C_{2}
\end{array}\right]\left[\begin{array}{c}
V_{1} \\
V_{2} \\
V_{3} \\
V_{4} \\
V_{5}
\end{array}\right]=\left[\begin{array}{c}
V_{s} \\
0 \\
0 \\
\frac{A_{v} V_{i}}{R_{o}} \\
0
\end{array}\right]
$$


(Step 4)

$$
\left[\begin{array}{ccccc}
1 & 0 & 0 & 0 & 0 \\
-s C_{1} & \frac{1}{R_{i}}+\frac{1}{R_{3}}+s C_{1} & -\frac{1}{R_{i}} & 0 & 0 \\
-\frac{1}{R_{1}} & -\frac{1}{R_{i}} & \frac{1}{R_{i}}+\frac{1}{R_{1}}+\frac{1}{R_{2}} & -\frac{1}{R_{2}} & 0 \\
0 & 0+\frac{A_{v}}{R_{o}} & -\frac{1}{R_{2}}-\frac{A_{v}}{R_{o}} & \frac{1}{R_{o}}+\frac{1}{R_{2}}+s C_{2} & -s C_{2} \\
-\frac{1}{R_{4}} & 0 & 0 & -s C_{2} & \frac{1}{R_{4}}+s C_{2}
\end{array}\right]\left[\begin{array}{c}
V_{1} \\
V_{2} \\
V_{3} \\
V_{4} \\
V_{5}
\end{array}\right]=\left[\begin{array}{c}
V_{s} \\
0 \\
0 \\
0 \\
0
\end{array}\right]
$$

(Step 5)

$$
\left[\begin{array}{ccccc}
1 & 0 & 0 & 0 & 0 \\
-s C_{1} & 0+\frac{1}{R_{3}}+s C_{1} & 0 & 0 & 0 \\
-\frac{1}{R_{1}} & 0 & 0+\frac{1}{R_{1}}+\frac{1}{R_{2}} & -\frac{1}{R_{2}} & 0 \\
0 & 0+\frac{A_{v}}{R_{o}} & 0-\frac{A_{v}}{R_{o}} & \frac{1}{R_{o}}+0 & -s C_{2} \\
-\frac{1}{R_{4}} & 0 & 0 & -s C_{2} & \frac{1}{R_{4}}+s C_{2}
\end{array}\right]\left[\begin{array}{c}
V_{1} \\
V_{2} \\
V_{3} \\
V_{4} \\
V_{5}
\end{array}\right]=\left[\begin{array}{c}
V_{s} \\
0 \\
0 \\
0 \\
0
\end{array}\right]
$$

(Step 6)

$$
\left[\begin{array}{ccccc}
1 & 0 & 0 & 0 & 0 \\
-s C_{1} & \frac{1}{R_{3}}+s C_{1} & 0 & 0 & 0 \\
-\frac{1}{R_{1}} & 0 & \frac{1}{R_{1}}+\frac{1}{R_{2}} & -\frac{1}{R_{2}} & 0 \\
0 & 1 & -1 & 0 & 0 \\
-\frac{1}{R_{4}} & 0 & 0 & -s C_{2} & \frac{1}{R_{4}}+s C_{2}
\end{array}\right]\left[\begin{array}{c}
V_{1} \\
V_{2} \\
V_{3} \\
V_{4} \\
V_{5}
\end{array}\right]=\left[\begin{array}{c}
V_{s} \\
0 \\
0 \\
0 \\
0
\end{array}\right]
$$


(Step 7)

$$
\left[\begin{array}{cc}
\frac{1}{R_{3}}+s C_{1} & 0 \\
\left(\frac{1}{R_{1}}+\frac{1}{R_{2}}\right)\left(-s R_{2} C_{2}\right) & \frac{1}{R_{4}}+s C_{2}
\end{array}\right]\left[\begin{array}{c}
V_{2}-V_{1} \\
V_{5}
\end{array}\right]=\left[\begin{array}{c}
-\frac{V_{s}}{R_{3}} \\
V_{s}\left(\frac{1}{R_{4}}+s C_{2}\right)
\end{array}\right]
$$

(Step 8)

$$
\begin{gathered}
\frac{V_{5}}{V_{s}}=\frac{s^{2}+\frac{1}{R^{2} C^{2}}}{s^{2}+s\left(\frac{2}{R C}\right)+\frac{1}{R^{2} C^{2}}} \equiv \frac{s^{2}+\omega_{0}^{2}}{s^{2}+s\left(\frac{\omega_{0}}{Q}\right)+\omega_{0}^{2}} \\
\omega_{0} \equiv \frac{1}{R C}, Q \equiv \frac{1}{2}
\end{gathered}
$$

\subsection{Low-pass filter}

A low-pass filter is an electronic filtering element that passes low-frequency signals and attenuates the amplitudes of signals with frequencies higher than a cutoff frequency. ${ }^{(11)}$ Consider the well-known low-pass filter with the operational amplifier ${ }^{(15)}$ shown in Fig. 12. The low-pass filter property can be easily obtained by the procedures of the proposed effective approach as follows, where Fig. 13 is the equivalent circuit of the filter in Fig. 12.

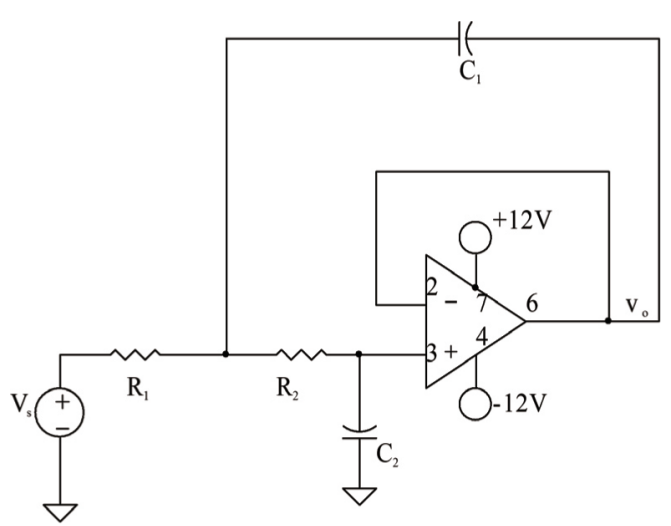

Fig. 12. Low-pass filter. 
(Step 1)

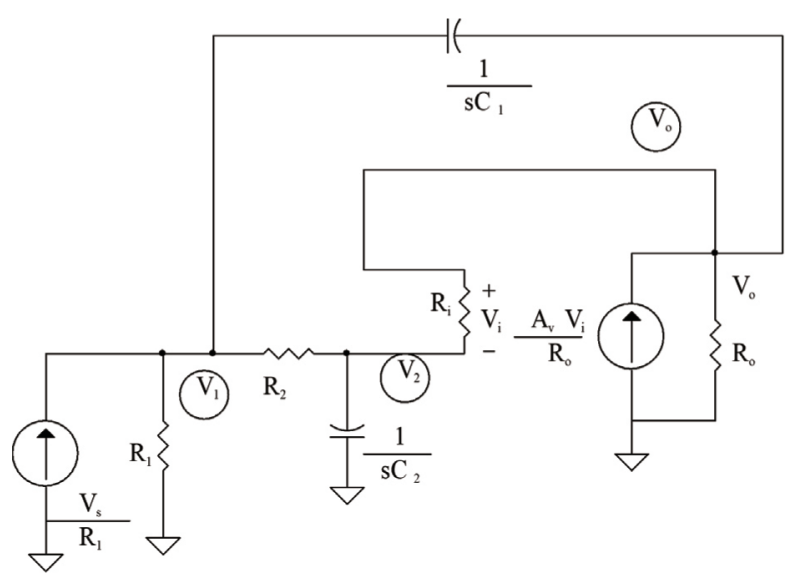

Fig. 13. Equivalent circuit of the filter in Fig. 12.

(Step 2)

$$
\left[\begin{array}{ccc}
\frac{1}{R_{1}}+\frac{1}{R_{2}}+s C_{1} & -\frac{1}{R_{2}} & -s C_{1} \\
-\frac{1}{R_{2}} & \frac{1}{R_{2}}+s C_{2}+\frac{1}{R_{i}} & -\frac{1}{R_{i}} \\
-s C_{1} & -\frac{1}{R_{i}} & \frac{1}{R_{i}}+s C_{1}+\frac{1}{R_{o}}
\end{array}\right]\left[\begin{array}{c}
V_{1} \\
V_{2} \\
V_{o}
\end{array}\right]=\left[\begin{array}{c}
\frac{V_{s}}{R_{1}} \\
0 \\
\frac{A_{v} V_{i}}{R_{o}}
\end{array}\right]
$$

(Step 3) and (Step 4)

$$
\left[\begin{array}{ccc}
\frac{1}{R_{1}}+\frac{1}{R_{2}}+s C_{1} & -\frac{1}{R_{2}} & -s C_{1} \\
-\frac{1}{R_{2}} & \frac{1}{R_{2}}+s C_{2}+\frac{1}{R_{i}} & -\frac{1}{R_{i}} \\
-s C_{1} & -\frac{1}{R_{i}}+\frac{A_{v}}{R_{o}} & \frac{1}{R_{i}}+s C_{1}+\frac{1}{R_{o}}-\frac{A_{v}}{R_{o}}
\end{array}\right]\left[\begin{array}{c}
V_{1} \\
V_{2} \\
V_{o}
\end{array}\right]=\left[\begin{array}{c}
\frac{V_{s}}{R_{1}} \\
0 \\
0
\end{array}\right]
$$

(Step 5)

$$
\left[\begin{array}{ccc}
\frac{1}{R_{1}}+\frac{1}{R_{2}}+s C_{1} & -\frac{1}{R_{2}} & -s C_{1} \\
-\frac{1}{R_{2}} & \frac{1}{R_{2}}+s C_{2}+0 & 0 \\
-s C_{1} & 0+\frac{A_{v}}{R_{o}} & 0+s C_{1}+\frac{1}{R_{o}}-\frac{A_{v}}{R_{o}}
\end{array}\right]\left[\begin{array}{c}
V_{1} \\
V_{2} \\
V_{o}
\end{array}\right]=\left[\begin{array}{c}
\frac{V_{s}}{R_{1}} \\
0 \\
0
\end{array}\right]
$$


(Step 6)

$$
\left[\begin{array}{ccc}
\frac{1}{R_{1}}+\frac{1}{R_{2}}+s C_{1} & -\frac{1}{R_{2}} & -s C_{1} \\
-\frac{1}{R_{2}} & \frac{1}{R_{2}}+s C_{2} & 0 \\
0 & 1 & -1
\end{array}\right]\left[\begin{array}{c}
V_{1} \\
V_{2} \\
V_{o}
\end{array}\right]=\left[\begin{array}{c}
\frac{V_{s}}{R_{1}} \\
0 \\
0
\end{array}\right]
$$

(Step 7)

$$
\left[\begin{array}{ccc}
\frac{1}{R_{1}}+\frac{1}{R_{2}}+s C_{1} & -\frac{1}{R_{2}} & -s C_{1}-\frac{1}{R_{2}} \\
-\frac{1}{R_{2}} & \frac{1}{R_{2}}+s C_{2} & \frac{1}{R_{2}}+s C_{2} \\
0 & 1 & 0
\end{array}\right]\left[\begin{array}{c}
V_{1} \\
V_{2}-V_{o} \\
V_{o}
\end{array}\right]=\left[\begin{array}{c}
\frac{V_{s}}{R_{1}} \\
0 \\
0
\end{array}\right]
$$

(Step 8)

$$
\begin{gathered}
\frac{V_{o}}{V_{s}}=\frac{\frac{1}{R_{1} R_{2} C_{1} C_{2}}}{s^{2}+s\left(\frac{1}{R_{1} C_{1}}+\frac{1}{R_{2} C_{1}}\right)+\frac{1}{R_{1} R_{2} C_{1} C_{2}}} \equiv \frac{n_{0}}{s^{2}+s\left(\frac{\omega_{0}}{Q}\right)+\omega_{0}^{2}} \\
f_{0}=\frac{1}{2 \pi \sqrt{R_{1} R_{2} C_{1} C_{2}}} \\
n_{0}=\frac{1}{R_{1} R_{2} C_{1} C_{2}} \\
\frac{\omega_{0}}{Q}=\frac{1}{R_{1} C_{1}}+\frac{1}{R_{2} C_{1}}
\end{gathered}
$$

\subsection{High-pass filter}

A high-pass filter is an electronic filtering device that passes high-frequency signals but attenuates the amplitudes of signals with frequencies lower than a cutoff frequency. ${ }^{(6)}$ Consider the well-known high-pass filter with the operational amplifier shown in Fig. 14. ${ }^{(21)}$ The procedures of the proposed effective approach are utilized to obtain the high-pass filter property as follows, where Fig. 15 is the equivalent circuit of the high-pass filter in Fig. 14. 


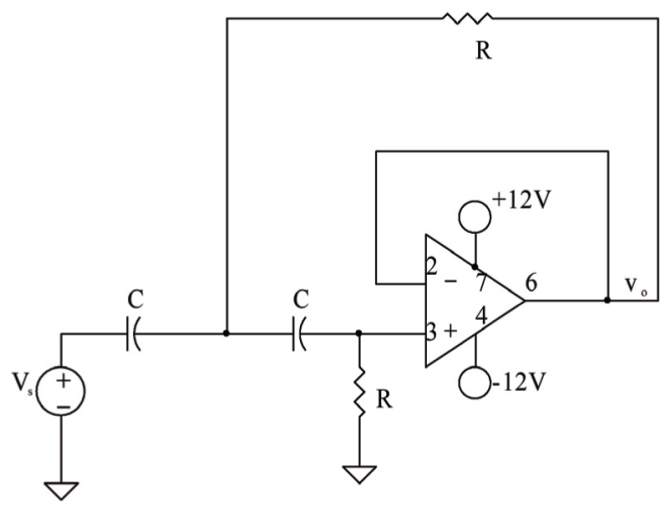

Fig. 14. High-pass filter.

(Step 1)

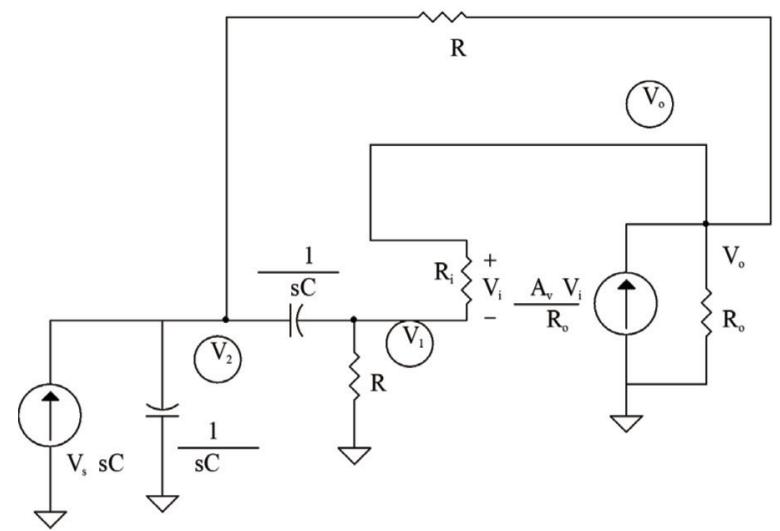

Fig. 15. Equivalent circuit of the high-pass filter in Fig. 14.

(Step 2)

$$
\left[\begin{array}{ccc}
\frac{1}{R}+s C+\frac{1}{R_{i}} & -s C & -\frac{1}{R_{i}} \\
-s C & \frac{1}{R}+2 s C & -\frac{1}{R} \\
-\frac{1}{R_{i}} & -\frac{1}{R} & \frac{1}{R_{i}}+\frac{1}{R}+\frac{1}{R_{o}}
\end{array}\right]\left[\begin{array}{l}
V_{1} \\
V_{2} \\
V_{o}
\end{array}\right]=\left[\begin{array}{c}
0 \\
V_{s}(s C) \\
\frac{A_{v} V_{i}}{R_{o}}
\end{array}\right]
$$


(Step 3) and (Step 4)

$$
\left[\begin{array}{ccc}
\frac{1}{R}+s C+\frac{1}{R_{i}} & -s C & -\frac{1}{R_{i}} \\
-s C & \frac{1}{R}+2 s C & -\frac{1}{R} \\
-\frac{1}{R_{i}}+\frac{A_{v}}{R_{o}} & -\frac{1}{R} & \frac{1}{R_{i}}+\frac{1}{R}+\frac{1}{R_{o}}-\frac{A_{v}}{R_{o}}
\end{array}\right]\left[\begin{array}{c}
V_{1} \\
V_{2} \\
V_{o}
\end{array}\right]=\left[\begin{array}{c}
0 \\
V_{s}(s C) \\
0
\end{array}\right]
$$

(Step 5)

$$
\left[\begin{array}{ccc}
\frac{1}{R}+s C+0 & -s C & 0 \\
-s C & \frac{1}{R}+2 s C & -\frac{1}{R} \\
0+\frac{A_{v}}{R_{o}} & -\frac{1}{R} & 0+\frac{1}{R}+\frac{1}{R_{o}}-\frac{A_{v}}{R_{o}}
\end{array}\right]\left[\begin{array}{c}
V_{1} \\
V_{2} \\
V_{o}
\end{array}\right]=\left[\begin{array}{c}
0 \\
V_{s}(s C) \\
0
\end{array}\right]
$$

(Step 6)

$$
\left[\begin{array}{ccc}
\frac{1}{R}+s C & -s C & 0 \\
-s C & \frac{1}{R}+2 s C & -\frac{1}{R} \\
1 & -\frac{1}{R} & -1
\end{array}\right]\left[\begin{array}{l}
V_{1} \\
V_{2} \\
V_{o}
\end{array}\right]=\left[\begin{array}{c}
0 \\
V_{s}(s C) \\
0
\end{array}\right]
$$

(Step 7)

$$
\left[\begin{array}{ccc}
\frac{1}{R}+s C & -s C & \frac{1}{R}+s C \\
-s C & \frac{1}{R}+2 s C & -\frac{1}{R}-s C \\
1 & 0 & 0
\end{array}\right]\left[\begin{array}{c}
V_{1}-V_{o} \\
V_{2} \\
V_{o}
\end{array}\right]=\left[\begin{array}{c}
0 \\
V_{s}(s C) \\
0
\end{array}\right]
$$

(Step 8)

$$
\frac{V_{o}}{V_{s}}=\frac{s^{2}}{s^{2}+s\left(\frac{2}{R C}\right)+\frac{1}{R^{2} C^{2}}}=\frac{n_{2} s^{2}}{s^{2}+s\left(\frac{\omega_{0}}{Q}\right)+\omega_{0}^{2}}
$$




$$
\begin{gathered}
f_{0}=\frac{1}{2 \pi R C} \\
Q=1 / 2
\end{gathered}
$$

\subsection{Non-inverting amplifier}

A non-inverting amplifier is a useful device that allows us to change the scale of a signal to any voltage range by adjusting the external resistors accordingly. ${ }^{(21)}$ Consider the wellknown non-inverting amplifier with the operational amplifier shown in Fig. 16. Applying the procedures of the proposed effective approach gives the desired non-inverting amplifier characteristics as follows, where Fig. 17 is the equivalent circuit of the non-inverting amplifier.

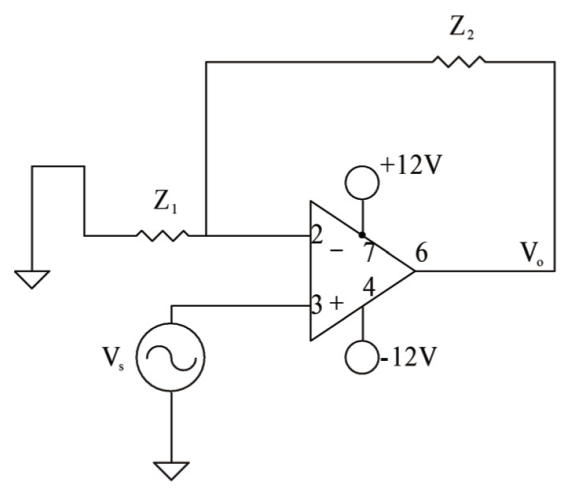

Fig. 16. Non-inverting amplifier.

(Step 1)

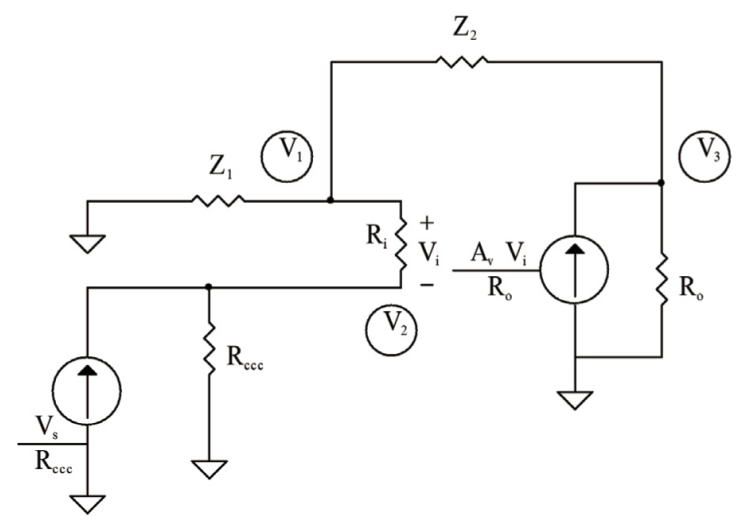

Fig. 17. Equivalent circuit of the non-inverting amplifier. 
(Step 2)

$$
\left[\begin{array}{ccc}
\frac{1}{Z_{1}}+\frac{1}{R_{i}}+\frac{1}{Z_{2}} & -\frac{1}{R_{i}} & -\frac{1}{Z_{2}} \\
-\frac{1}{R_{i}} & \frac{1}{R_{c c c}}+\frac{1}{R_{i}} & 0 \\
-\frac{1}{Z_{2}} & 0 & \frac{1}{Z_{2}}+\frac{1}{R_{o}}
\end{array}\right]\left[\begin{array}{l}
V_{1} \\
V_{2} \\
V_{3}
\end{array}\right]=\left[\begin{array}{c}
0 \\
\frac{V_{s}}{R_{c c c}} \\
\frac{A_{v} V_{i}}{R_{o}}
\end{array}\right]
$$

(Step 3)

$$
\left[\begin{array}{ccc}
\frac{1}{Z_{1}}+\frac{1}{R_{i}}+\frac{1}{Z_{2}} & -\frac{1}{R_{i}} & -\frac{1}{Z_{2}} \\
0 & 1 & 0 \\
-\frac{1}{Z_{2}} & 0 & \frac{1}{Z_{2}}+\frac{1}{R_{o}}
\end{array}\right]\left[\begin{array}{c}
V_{1} \\
V_{2} \\
V_{3}
\end{array}\right]=\left[\begin{array}{c}
0 \\
V_{s} \\
\frac{A_{v} V_{i}}{R_{o}}
\end{array}\right]
$$

(Step 4)

$$
\left[\begin{array}{ccc}
\frac{1}{Z_{1}}+\frac{1}{R_{i}}+\frac{1}{Z_{2}} & -\frac{1}{R_{i}} & -\frac{1}{Z_{2}} \\
0 & 1 & 0 \\
-\frac{1}{Z_{2}}-\frac{A_{v}}{R_{o}} & +\frac{A_{v}}{R_{o}} & \frac{1}{Z_{2}}+\frac{1}{R_{o}}
\end{array}\right]\left[\begin{array}{c}
V_{1} \\
V_{2} \\
V_{3}
\end{array}\right]=\left[\begin{array}{c}
0 \\
V_{s} \\
0
\end{array}\right]
$$

(Step 5)

$$
\left[\begin{array}{ccc}
\frac{1}{Z_{1}}+0+\frac{1}{Z_{2}} & 0 & -\frac{1}{Z_{2}} \\
0 & 1 & 0 \\
-\frac{A_{v}}{R_{o}} & +\frac{A_{v}}{R_{o}} & \frac{1}{R_{o}}
\end{array}\right]\left[\begin{array}{c}
V_{1} \\
V_{2} \\
V_{3}
\end{array}\right]=\left[\begin{array}{c}
0 \\
V_{s} \\
0
\end{array}\right]
$$

(Step 6)

$$
\left[\begin{array}{ccc}
\frac{1}{Z_{1}}+\frac{1}{Z_{2}} & -0 & -\frac{1}{Z_{2}} \\
0 & 1 & 0 \\
-1 & 1 & 0
\end{array}\right]\left[\begin{array}{c}
V_{1} \\
V_{2} \\
V_{3}
\end{array}\right]=\left[\begin{array}{c}
0 \\
V_{s} \\
0
\end{array}\right]
$$


(Step 7) and (Step 8)

$$
V_{o}=V_{3}=\left(\frac{Z_{1}+Z_{2}}{Z_{1}}\right) V_{s}
$$

\subsection{Voltage follower amplifier}

If the signal is transferred without any changes, the amplifier is a unity gain buffer known as a voltage follower because the output voltage follows the input signal. ${ }^{(16)}$ Consider the wellknown voltage follower amplifier with an operational amplifier shown in Fig. 18. The property of the voltage follower amplifier can be obtained by the procedures of the proposed effective algorithm as follows, where Fig. 19 is the equivalent circuit of Fig. 18.

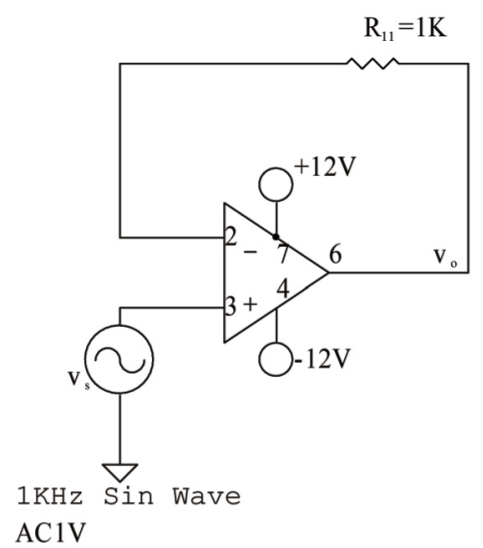

Fig. 18. Voltage follower.

(Step 1)

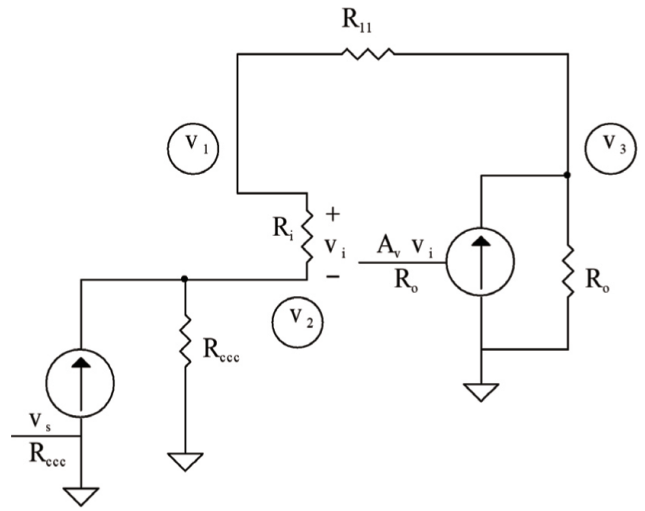

Fig. 19. Equivalent circuit of Fig. 18. 
(Step 2)

$$
\left[\begin{array}{ccc}
\frac{1}{R_{11}}+\frac{1}{R_{i}} & -\frac{1}{R_{i}} & -\frac{1}{R_{11}} \\
-\frac{1}{R_{i}} & \frac{1}{R_{i}}+\frac{1}{R_{c c c}} & 0 \\
-\frac{1}{R_{11}} & 0 & \frac{1}{R_{11}}+\frac{1}{R_{o}}
\end{array}\right]\left[\begin{array}{l}
v_{1} \\
v_{2} \\
v_{3}
\end{array}\right]=\left[\begin{array}{c}
0 \\
\frac{v_{s}}{R_{c c c}} \\
\frac{A_{v} v_{i}}{R_{o}}
\end{array}\right]
$$

(Step 3)

$$
\left[\begin{array}{ccc}
\frac{1}{R_{11}}+\frac{1}{R_{i}} & -\frac{1}{R_{i}} & -\frac{1}{R_{11}} \\
0 & 1 & 0 \\
-\frac{1}{R_{11}} & 0 & \frac{1}{R_{11}}+\frac{1}{R_{o}}
\end{array}\right]\left[\begin{array}{c}
v_{1} \\
v_{2} \\
v_{3}
\end{array}\right]=\left[\begin{array}{c}
0 \\
v_{s} \\
\frac{A_{v} v_{i}}{R_{o}}
\end{array}\right]
$$

(Step 4)

$$
\left[\begin{array}{ccc}
\frac{1}{R_{11}}+\frac{1}{R_{i}} & -\frac{1}{R_{i}} & -\frac{1}{R_{11}} \\
0 & 1 & 0 \\
-\frac{1}{R_{11}}-\frac{A_{v}}{R_{o}} & +\frac{A_{v}}{R_{o}} & \frac{1}{R_{11}}+\frac{1}{R_{o}}
\end{array}\right]\left[\begin{array}{c}
v_{1} \\
v_{2} \\
v_{3}
\end{array}\right]=\left[\begin{array}{c}
0 \\
v_{s} \\
0
\end{array}\right]
$$

(Step 5)

$$
\left[\begin{array}{ccc}
\frac{1}{R_{11}}+0 & 0 & -\frac{1}{R_{11}} \\
0 & 1 & 0 \\
-\frac{1}{R_{11}}-\frac{A_{v}}{R_{o}} & +\frac{A_{v}}{R_{o}} & 0+\frac{1}{R_{o}}
\end{array}\right]\left[\begin{array}{l}
v_{1} \\
v_{2} \\
v_{3}
\end{array}\right]=\left[\begin{array}{c}
0 \\
v_{s} \\
0
\end{array}\right]
$$

(Step 6)

$$
\left[\begin{array}{ccc}
\frac{1}{R_{11}} & 0 & -\frac{1}{R_{11}} \\
0 & 1 & 0 \\
-1 & +1 & 0
\end{array}\right]\left[\begin{array}{c}
v_{1} \\
v_{2} \\
v_{3}
\end{array}\right]=\left[\begin{array}{c}
0 \\
v_{s} \\
0
\end{array}\right]
$$


(Step 7) and (Step 8)

$$
v_{o}=v_{3}=\frac{-v_{s}\left(1 / R_{11}\right)}{-\left(1 / R_{11}\right)}=v_{s}
$$

\subsection{DC-level adjusting amplifier}

Consider the well-known DC-level adjusting amplifier with an operational amplifier shown in Fig. 20. ${ }^{(21)}$ Using the procedures of the proposed effective approach yields the DC-level adjusting amplifier performance as follows, where Fig. 21 is the equivalent circuit of Fig. 20.

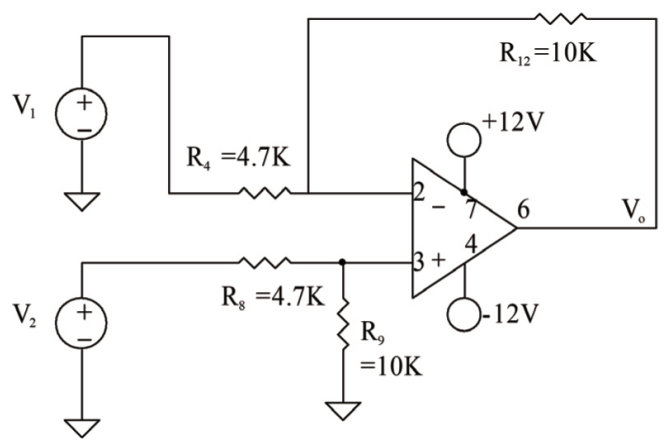

Fig. 20. DC-level adjusting amplifier.

(Step 1)

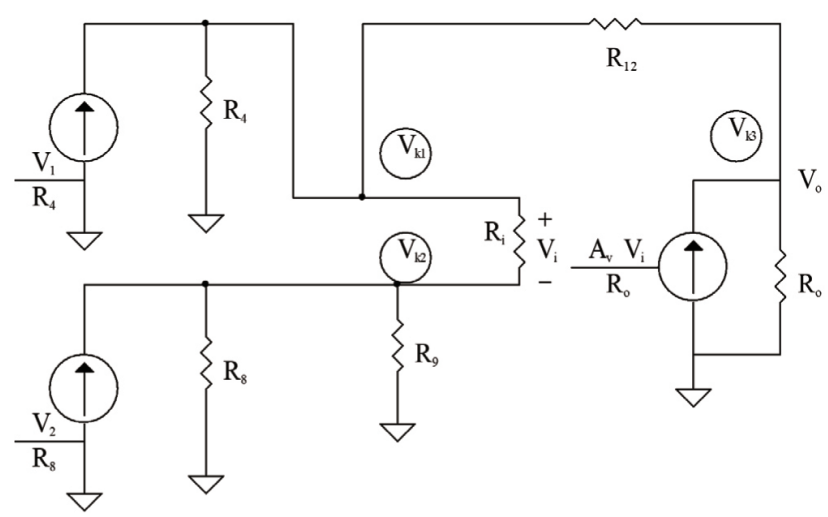

Fig. 21. Equivalent circuit of Fig. 20. 
(Step 2)

$$
\left[\begin{array}{ccc}
\frac{1}{R_{4}}+\frac{1}{R_{12}}+\frac{1}{R_{i}} & -\frac{1}{R_{i}} & -\frac{1}{R_{12}} \\
-\frac{1}{R_{i}} & \frac{1}{R_{8}}+\frac{1}{R_{9}}+\frac{1}{R_{i}} & 0 \\
-\frac{1}{R_{12}} & 0 & \frac{1}{R_{12}}+\frac{1}{R_{o}}
\end{array}\right]\left[\begin{array}{l}
V_{k 1} \\
V_{k 2} \\
V_{k 3}
\end{array}\right]=\left[\begin{array}{c}
\frac{V_{1}}{R_{4}} \\
\frac{V_{2}}{R_{8}} \\
\frac{A_{v} V_{i}}{R_{o}}
\end{array}\right]
$$

(Step 3) and (Step 4)

$$
\left[\begin{array}{ccc}
\frac{1}{R_{4}}+\frac{1}{R_{12}}+\frac{1}{R_{i}} & -\frac{1}{R_{i}} & -\frac{1}{R_{12}} \\
-\frac{1}{R_{i}} & \frac{1}{R_{8}}+\frac{1}{R_{9}}+\frac{1}{R_{i}} & 0 \\
-\frac{1}{R_{12}}-\frac{A_{v}}{R_{o}} & +\frac{A_{v}}{R_{o}} & \frac{1}{R_{12}}+\frac{1}{R_{o}}
\end{array}\right]\left[\begin{array}{c}
V_{k 1} \\
V_{k 2} \\
V_{k 3}
\end{array}\right]=\left[\begin{array}{c}
\frac{V_{1}}{R_{4}} \\
\frac{V_{2}}{R_{8}} \\
0
\end{array}\right]
$$

(Step 5)

$$
\left[\begin{array}{ccc}
\frac{1}{R_{4}}+\frac{1}{R_{12}}+0 & 0 & -\frac{1}{R_{12}} \\
0 & \frac{1}{R_{8}}+\frac{1}{R_{9}}+0 & 0 \\
-\frac{1}{R_{12}}-\frac{A_{v}}{R_{o}} & +\frac{A_{v}}{R_{o}} & 0+\frac{1}{R_{o}}
\end{array}\right]\left[\begin{array}{c}
V_{k 1} \\
V_{k 2} \\
V_{k 3}
\end{array}\right]=\left[\begin{array}{c}
\frac{V_{1}}{R_{4}} \\
\frac{V_{2}}{R_{8}} \\
0
\end{array}\right]
$$

(Step 6)

$$
\left[\begin{array}{ccc}
\frac{1}{R_{4}}+\frac{1}{R_{12}} & 0 & -\frac{1}{R_{12}} \\
0 & \frac{1}{R_{8}}+\frac{1}{R_{9}} & 0 \\
-1 & +1 & \frac{1}{R_{o}}
\end{array}\right]\left[\begin{array}{c}
V_{k 1} \\
V_{k 2} \\
V_{k 3}
\end{array}\right]=\left[\begin{array}{c}
\frac{V_{1}}{R_{4}} \\
\frac{V_{2}}{R_{8}} \\
0
\end{array}\right]
$$


(Step 7) and (Step 8)

$$
V_{o}=V_{k 3}=V_{1}\left(-\frac{R_{12}}{R_{4}}\right)+V_{2}\left(\frac{1+\frac{R_{12}}{R_{4}}}{1+\frac{R_{8}}{R_{9}}}\right)
$$

\subsection{Authors' invented adder}

Consider the authors' newly invented adder with the significant advantage of arbitrary numbers of inverting ends and non-inverting ends as shown in Fig. 22. The procedures of the proposed effective approach are employed to obtain the performance of the authors' invented adder as follows, where Fig. 23 is the equivalent circuit of the authors' invented adder in Fig. 22.

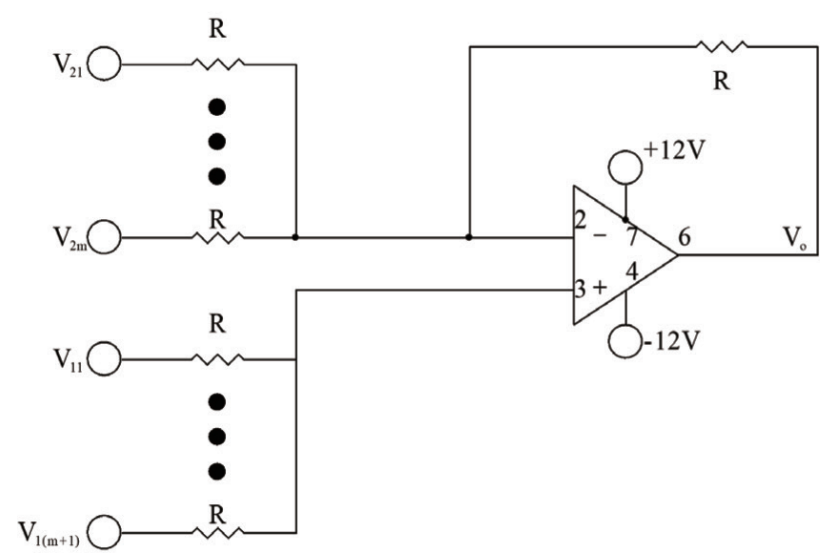

Fig. 22. Authors' invented adder.

(Step 1)

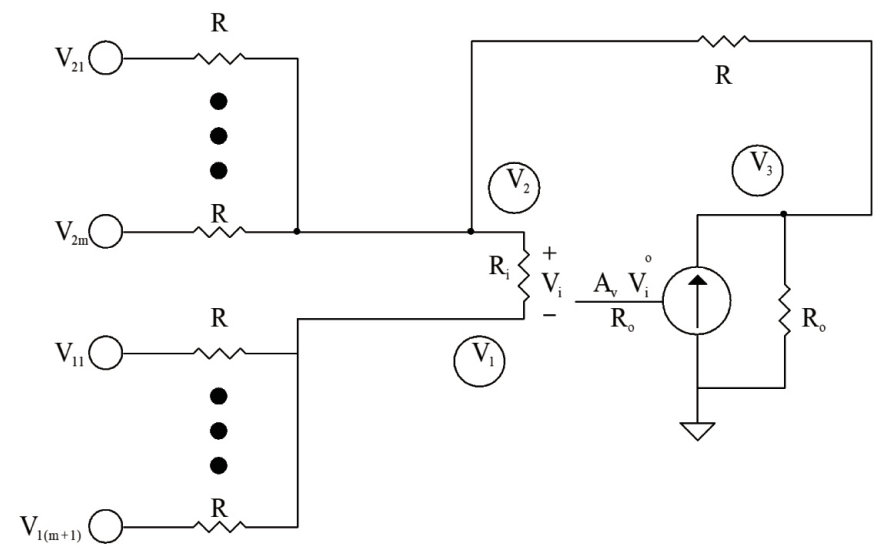

Fig. 23. Equivalent circuit of the authors' invented adder in Fig. 22. 
(Step 2)

$$
\left[\begin{array}{ccc}
\frac{m+1}{R}+\frac{1}{R_{i}} & -\frac{1}{R_{i}} & 0 \\
-\frac{1}{R_{i}} & \frac{m+1}{R}+\frac{1}{R_{i}} & -\frac{1}{R} \\
0 & -\frac{1}{R} & \frac{1}{R}+\frac{1}{R_{o}}
\end{array}\right]\left[\begin{array}{l}
V_{1} \\
V_{2} \\
V_{3}
\end{array}\right]=\left[\begin{array}{c}
\frac{V_{11}+V_{12}+\ldots+V_{1(m+1)}}{R} \\
\frac{V_{21}+\ldots+V_{2 m}}{R} \\
\frac{A_{v} V_{i}}{R_{o}}
\end{array}\right]
$$

(Step 3)

$$
\left[\begin{array}{ccc}
\frac{m+1}{R}+\frac{1}{R_{i}} & -\frac{1}{R_{i}} & 0 \\
-\frac{1}{R_{i}} & \frac{m+1}{R}+\frac{1}{R_{i}} & -\frac{1}{R} \\
0+\frac{A_{v}}{R_{o}} & -\frac{1}{R}-\frac{A_{v}}{R_{o}} & \frac{1}{R}+\frac{1}{R_{o}}
\end{array}\right]\left[\begin{array}{l}
V_{1} \\
V_{2} \\
V_{3}
\end{array}\right]=\left[\begin{array}{c}
\frac{V_{11}+V_{12}+\ldots+V_{1(m+1)}}{R} \\
\frac{V_{21}+\ldots+V_{2 m}}{R} \\
0
\end{array}\right]
$$

(Step 4)

$$
\left[\begin{array}{ccc}
\frac{m+1}{R}+0 & 0 & 0 \\
0 & \frac{m+1}{R}+0 & -\frac{1}{R} \\
0+\frac{A_{v}}{R_{o}} & -\frac{1}{R}-\frac{A_{v}}{R_{o}} & \frac{1}{R}+\frac{1}{R_{o}}
\end{array}\right]\left[\begin{array}{l}
V_{1} \\
V_{2} \\
V_{3}
\end{array}\right]=\left[\begin{array}{c}
\frac{V_{11}+V_{12}+\ldots+V_{1(m+1)}}{R} \\
\frac{V_{21}+\ldots+V_{2 m}}{R} \\
0
\end{array}\right]
$$

(Step 5)

$$
\left[\begin{array}{ccc}
\frac{m+1}{R} & 0 & 0 \\
0 & \frac{m+1}{R} & -\frac{1}{R} \\
1 & -1 & 0
\end{array}\right]\left[\begin{array}{l}
V_{1} \\
V_{2} \\
V_{3}
\end{array}\right]=\left[\begin{array}{c}
\frac{V_{11}+V_{12}+\ldots+V_{1(m+1)}}{R} \\
\frac{V_{21}+\ldots+V_{2 m}}{R} \\
0
\end{array}\right]
$$


(Step 6)

$$
\left[\begin{array}{ccc}
\frac{m+1}{R} & 0 & 0 \\
\frac{m+1}{R} & 0 & -\frac{1}{R} \\
1 & -1 & 0
\end{array}\right]\left[\begin{array}{l}
V_{1} \\
V_{2} \\
V_{3}
\end{array}\right]=\left[\begin{array}{c}
\frac{V_{11}+V_{12}+\ldots+V_{1(m+1)}}{R} \\
\frac{V_{21}+\ldots+V_{2 m}}{R} \\
0
\end{array}\right]
$$

(Step 7)

$$
V_{o}=\left(V_{11}+\ldots+V_{1(m+1)}\right)-\left(V_{21}+\ldots+V_{2 m}\right)
$$

\section{Experiments on the Online Electrocardiograph Device}

This block diagram and hardware implementation of the online electrocardiograph device are shown in Figs. 24 and 25, respectively.

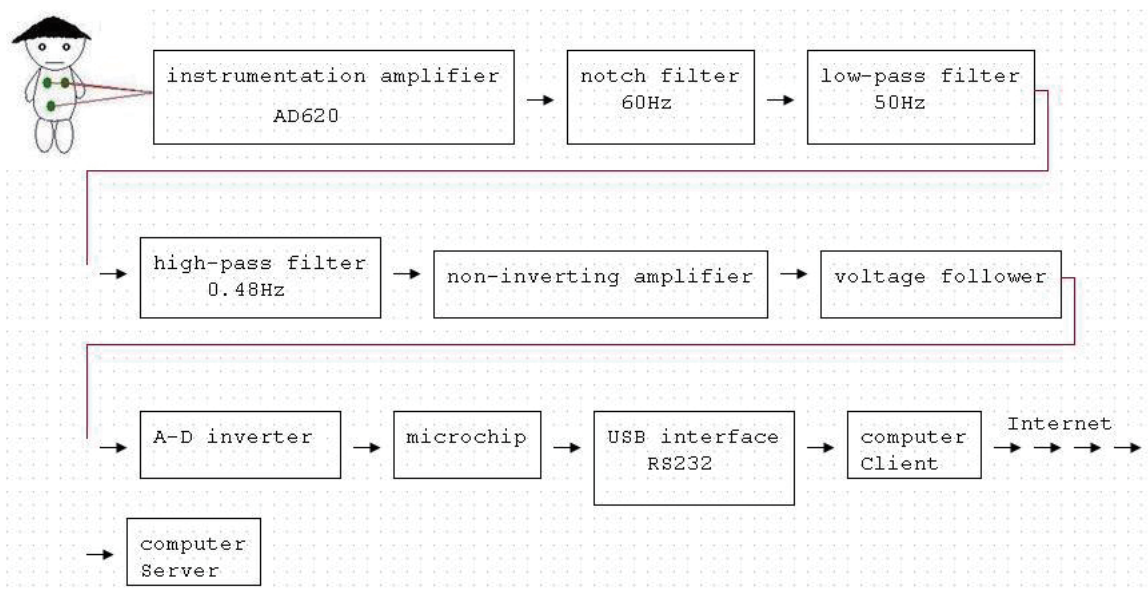

Fig. 24. (Color online) Block diagram of the online electrocardiograph device.

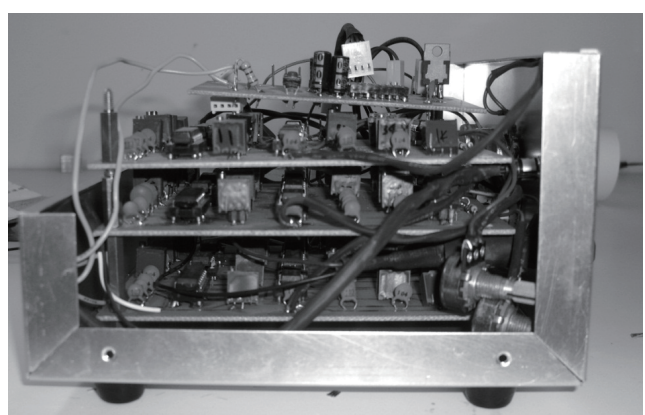

(a)

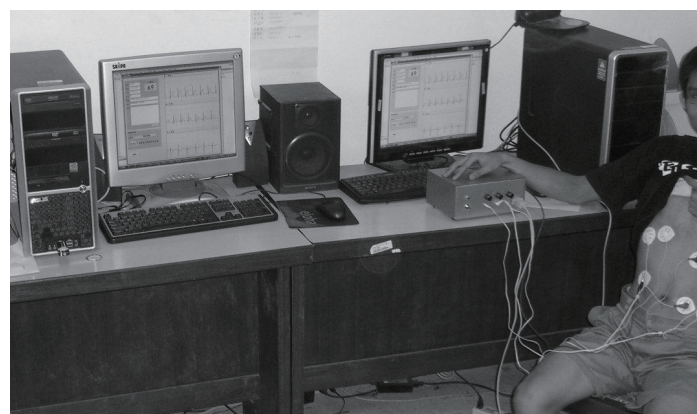

(b)

Fig. 25. Hardware implementation of the online electrocardiograph device. 
The first observation of the original ECG signal through the first-zone AD620 instrumentation amplifier is shown in Fig. 26. Figure 26 shows that the original ECG signal contains all low-frequency and high-frequency noises and power noise. In general, the electronic device is connected to a power source and thus the $60 \mathrm{~Hz}$ noise is inevitable. Thus, the original signal will generate the $60 \mathrm{~Hz}$ noise impact. Figure 27 clearly shows that the impact of the $60 \mathrm{~Hz}$ noise is completely eliminated by the second-zone $60 \mathrm{~Hz}$ notch filter. In general, the frequency of an ECG signal is approximately $50 \mathrm{~Hz}$. Therefore, in addition to the $60 \mathrm{~Hz}$ noise, many high-frequency noises need to be filtered, and so we include a third-zone low-pass filter to reduce high-frequency noises. The observation of the ECG signal after passing through the low-pass filter is shown in Fig. 28. As we take an ECG signal with a frequency of about 50 $\mathrm{Hz}$, the fourth-zone high-pass filter is designed with a cutoff frequency of $40 \mathrm{~Hz}$. The output wave of the ECG signal after passing through the high-pass filter is shown in Fig. 29. To cancel the general load effect of the ECG circuit, we should add a voltage follower to the overall circuit. The signal is then passed through the fifth-zone non-inverting amplifier and voltage follower. It is clear that the amplitude is significantly increased as a result from Figs. 30 and 31. To enable the utilization of internet transmission technology, we should transform the analog signal to a

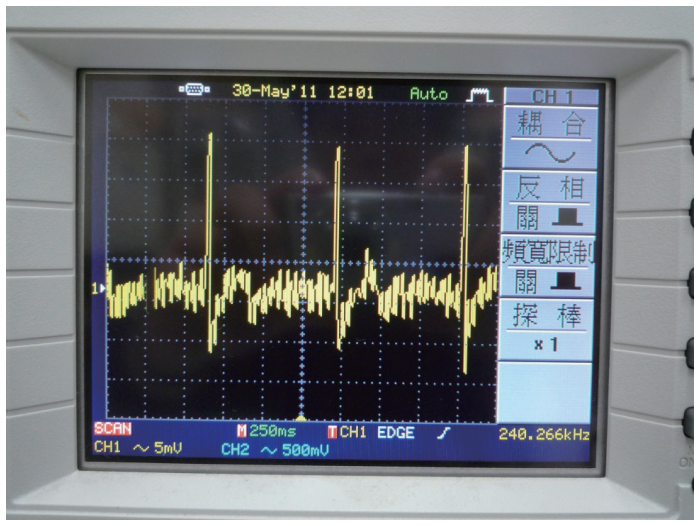

Fig. 26. (Color online) Measured wave at the output part for the AD620 instrumentation amplifier.

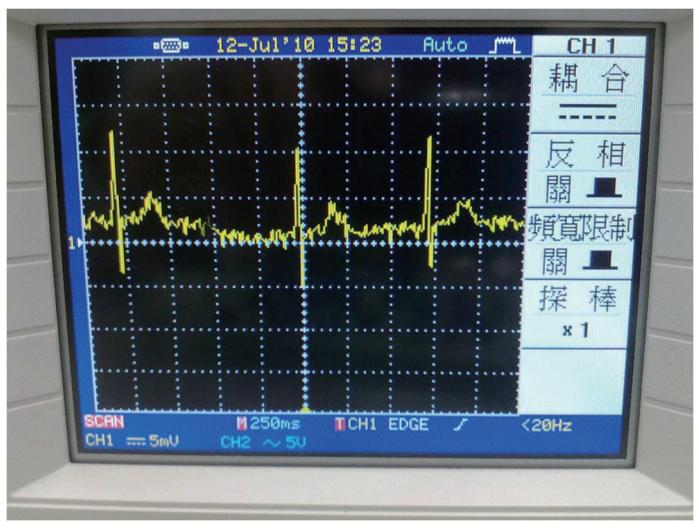

Fig. 28. (Color online) Measured wave after passing through the low-pass filter.

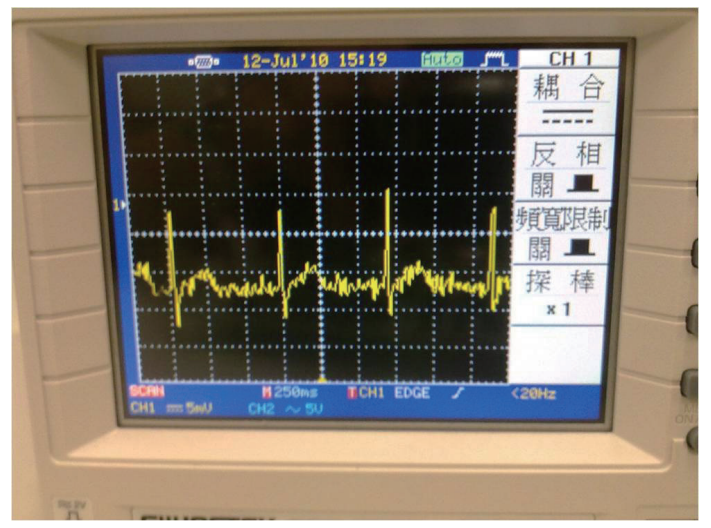

Fig. 27. (Color online) Measured wave after passing through the $60 \mathrm{~Hz}$ notch filter.

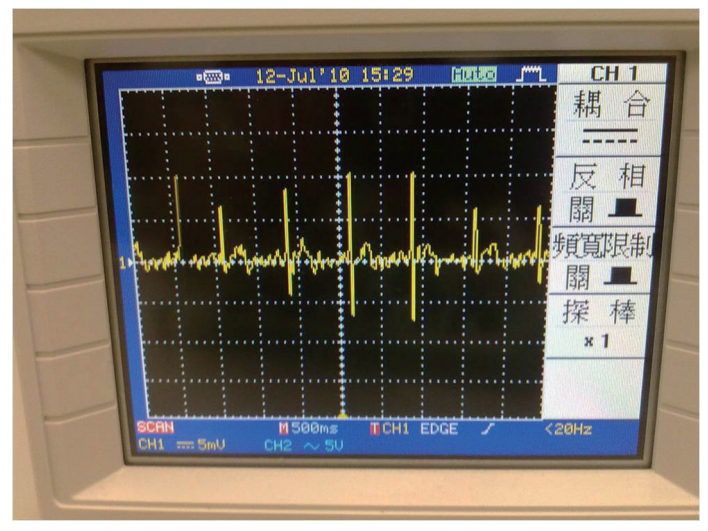

Fig. 29. (Color online) Measured wave after passing through the high-pass filter. 


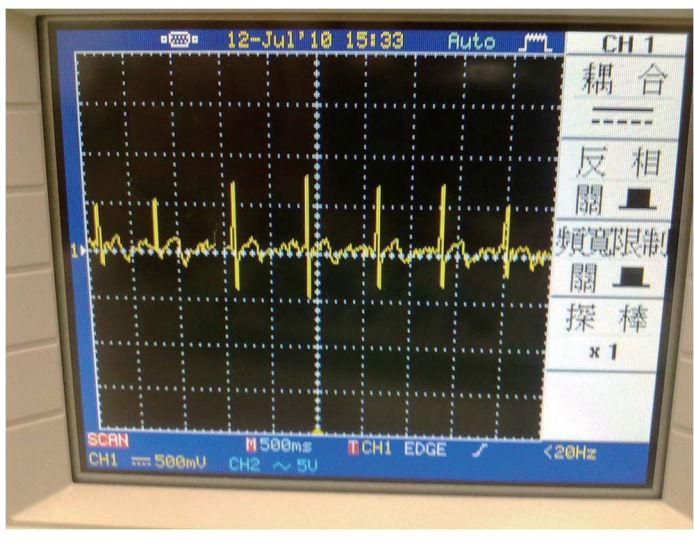

Fig. 30. (Color online) Measured wave after passing through the non-inverting amplifier.

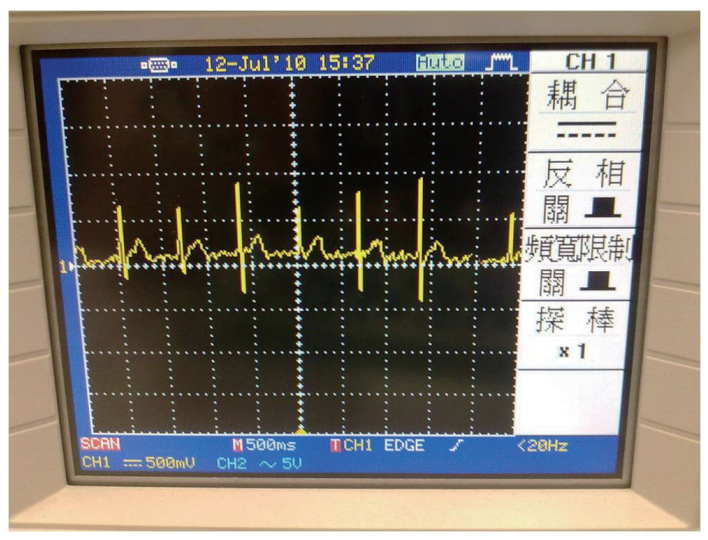

Fig. 31. (Color online) Measured wave after passing through the voltage-follower amplifier.

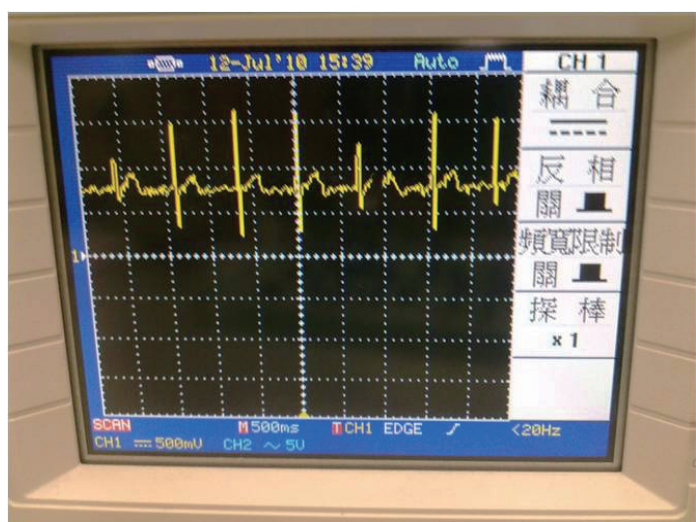

Fig. 32. (Color online) Measured wave after passing through the DC-level adjusting amplifier.

digital signal via an analog/digital (A/D) converter. To match the rated input voltage level of the A/D converter, we use an ADC0804 converter with a conversion range 0-5 V, and so we need to increase the ECG signal flow level by utilizing the sixth-zone DC-level adjusting amplifier with the authors' invented adder. From the comparison of Figs. 31 and 32, the ECG signal flow level is increased by $0.75 \mathrm{~V}$ and thus the ECG signal can pass through the A/D converter to the client computer shown in Fig. 33. Subsequently, we can pass the client's ECG signal to the hospital server shown in Fig. 33 by exploring internet transmission technology.

As a comparison with other existing methods, we demonstrate a Bessel low-pass filter that is mainly made up of an AD8618 quad op-amp. ${ }^{(14)}$ The transfer function shown in Ref. 14 is clearly incorrect in the non-ideal case with $A_{v}=-10^{4} \neq \infty, R_{i}=\infty$, and $R_{o}=0$. Moreover, the transfer functions of the AD8220 instrumentation and main AD8618 amplifers are incorrect in a non-ideal case, and thus the important amplitude-frequency and phase-frequency characteristic curves will not be exact. We show all the processes for deriving the transfer function for the Bessel low-pass filter in Fig. 34. 


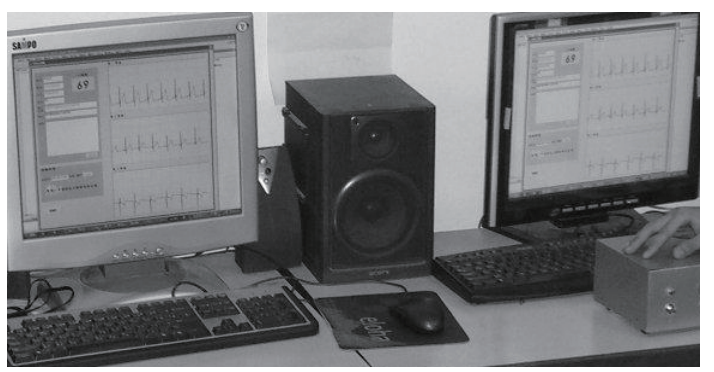

Fig. 33. Measured waves of client computer and hospital server used for the ECG signal.

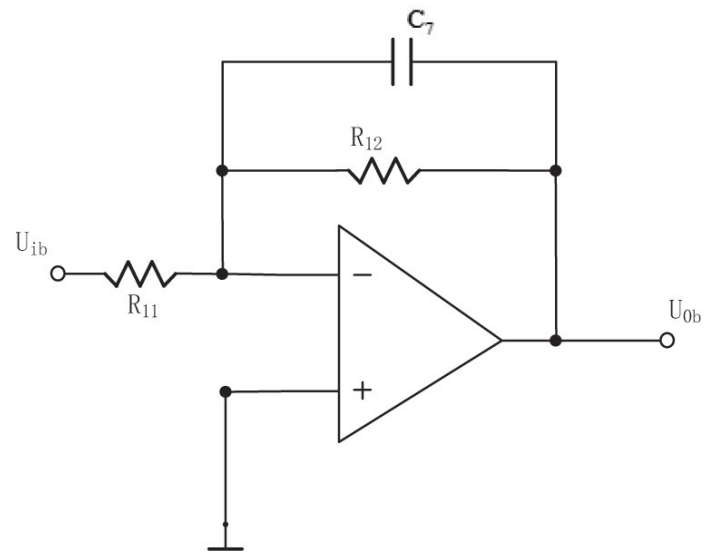

Fig. 34. Bessel low-pass filter.

(Step 1) The Bessel low-pass filter can be replaced with the operational amplifier equivalent circuit (Fig. 35).

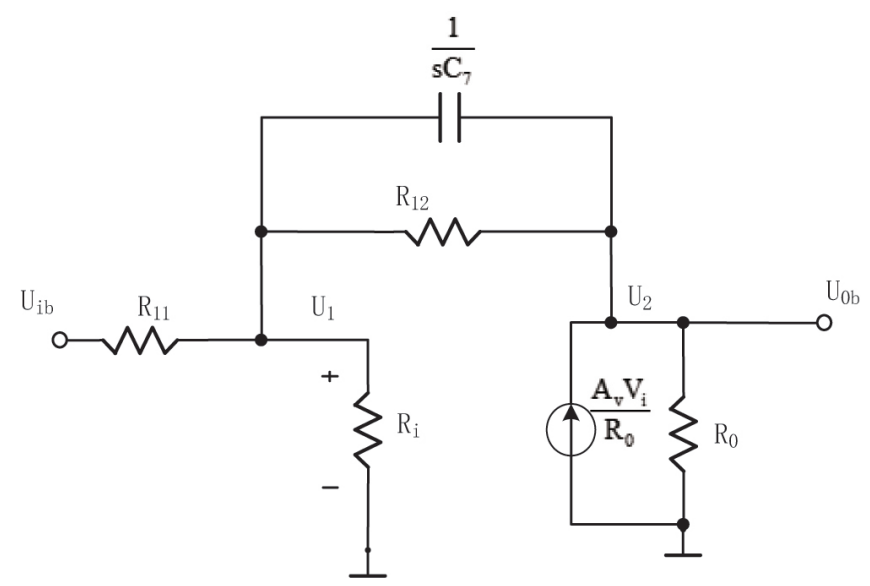

Fig. 35. Equivalent circuit of Fig. 24.

(Step 2) List the matrix node-voltage equations.

$$
\left[\begin{array}{cc}
\frac{1}{R_{11}}+\frac{1}{R_{12}}+s C_{7} & -\frac{1}{R_{12}}-s C_{7} \\
-\frac{1}{R_{12}}-s C_{7} & \frac{1}{R_{o}}+\frac{1}{R_{12}}+s C_{7}
\end{array}\right]\left[\begin{array}{c}
U_{1} \\
U_{2}
\end{array}\right]=\left[\begin{array}{c}
\frac{U_{i b}}{R_{11}} \\
\frac{A_{v} V_{i}}{R_{o}}
\end{array}\right]
$$

Solve the dependent source element by performing simple moving-term operations based on the equation $V_{i}=U_{1}$. 


$$
\left[\begin{array}{cc}
\frac{1}{R_{11}}+\frac{1}{R_{12}}+s C_{7} & -\frac{1}{R_{12}}-s C_{7} \\
-\frac{1}{R_{12}}-s C_{7}-\frac{A_{v}}{R_{o}} & \frac{1}{R_{o}}+\frac{1}{R_{12}}+s C_{7}
\end{array}\right]\left[\begin{array}{c}
U_{1} \\
U_{2}
\end{array}\right]=\left[\begin{array}{c}
\frac{U_{i b}}{R_{11}} \\
0
\end{array}\right] \leftarrow \times R_{o}
$$

Use our invented simple matrix operations and the L'Hospital rule to reduce the matrix equation.

$$
\left[\begin{array}{cc}
\frac{1}{R_{11}}+\frac{1}{R_{12}}+s C_{7} & -\frac{1}{R_{12}}-s C_{7} \\
-A_{v} & 1
\end{array}\right]\left[\begin{array}{c}
U_{1} \\
U_{2}
\end{array}\right]=\left[\begin{array}{c}
\frac{U_{i b}}{R_{11}} \\
0
\end{array}\right]
$$

(Step 3) Apply Cramer's rule to derive the transfer function.

$$
U_{2}(s)=U_{o b}(s)=\frac{\left(\frac{A_{v}}{R_{11}}\right) U_{i b}}{\left(\frac{1}{R_{11}}+\frac{1}{R_{12}}-\frac{A_{v}}{R_{12}}\right)+s\left(C_{7}-A_{v} C_{7}\right)}
$$

Thus, the frequency-domain transfer function is given by

$$
\frac{U_{o b}(j \omega)}{U_{i b}(j \omega)}=\frac{\left(\frac{A_{v}}{R_{11}}\right)}{\left(\frac{1}{R_{11}}+\frac{1}{R_{12}}-\frac{A_{v}}{R_{12}}\right)+j \omega\left(C_{7}-A_{v} C_{7}\right)} .
$$

It is clear that this equation is not equal to Eq. (7) of Ref. 14.

\section{Conclusions}

The proposed efficient algorithm, which is an alternative to using the virtual ground technique, Kirchhoff's voltage/current law, and traditional Thevenin approaches, of a complicated operational amplifier system combines the L'Hospital rule, fundamental circuit theory, and the simple matrix operations developed by the authors, enabling us to easily design an online electrocardiograph device. A theoretical investigation of the complicated operational amplifier system including instrumentation and frequency sensors has been presented and finally an online electrocardiograph device has been implemented using the proposed simple approach. Note that so far, the existing approaches have not provided a systematic matrix solution for complicated operational amplifier circuit systems. In the future, we will extend 
the complicated operational amplifier approach to the design of electronic measuring circuits, robust control systems, robotics control, and industrial applications.

\section{Acknowledgments}

This work was supported by Institute-level major scientific research and training projects (Lead Doctor special projects) of City College of Dongguan University of Technology by Grant No. 2017YZDYB01Z (Grant Title: Create a new theory and technology of electric circuits and electronics science based on Chen's electrical unifying approach). This work was also supported by City College of Dongguan University of Technology (Grant No.: 201813844009).

\section{References}

1 C. K. Alexander and M. N. O. Sadiku: Fundamentals of Electric Circuits (McGraw-Hill, New York, 2018).

2 A. Anvesha and M. S. Baghini: Proc. 14th Int. Symp. Quality Electronic Design (IEEE, 2013) 689-695.

3 C. C. Chen, T. L. Chien, Y. C. Chen, W. J. Lin, and S. H. Yang: Chaos Solitons Fractals 42 (2009) 2464. https:// doi.org/10.1016/j.chaos.2009.03.113

4 C. C. Chen: New Simple Chen's Electric Unifying Approach of Electronic Experiment (Lie Guan Press, Taiwan, 2010) (in Chinese).

5 T. L. Chien, C. J. Huang, Y. C. Chen, C. C. Chen, and C. W. Lin: Innovative Comput. Inf. Control Exp. Lett. Part B: Applications 2 (2011) 919.

6 Y. T. Chou and H. C. Lu: Trans. Microwave Theory Tech. 61 (2013) 2460.

7 D. R. Cunningham and J. A. Stuller: Basic Circuit Analysis (Houghton Mifflin, Boston, 1991).

8 R. C. Dorf and J. A. Svoboda: Introduction to Electric Circuits (John Wiley and Sons, New Jersey, 2006).

9 A. Ghaffari, E. A. M. Klumperink, and B. Nauta: IEEE J. Solid-State Circuits 48 (2013) 1370. https://doi. org/10.1109/JSSC.2013.2252521

10 P. E. Gray and C. L. Searle: Electronic Principles (Wiley, New York, 1969).

11 M. Kufa and Z. Raida: Electron. Lett. 49 (2013) 199. https://doi.org/10.1049/el.2012.3473

12 T. Le, S. Bukkapatnam, and R. Komanduri: IEEE Trans. Biomed. Eng. 60 (2013) 2350. https://doi.org/10.1109/ TBME.2013.2256423

13 M. Li, J. Zhao, W. Zhang, and R. Zheng: The 2nd Int. Conf. Information Science and Engineering (IClSE, 2010) (Hangzhou, 2010) 207-209.

14 Y. Li, X. Xu, Y. Liu, and Y. Tian: 2011 Int. Conf. Information Technology, Computer Engineering and Management Sciences (2011) 309-312.

15 J. Millman: Microelectronics: Digital and Analog Circuits and Systems (McGraw-Hill, New York, 1979).

16 K. Moustakas and S. Siskos: IEEE Int. Conf. Industrial Technology (2013) 961-965. https://doi.org/10.1109/ ICIT.2013.6505801

17 J. W. Nilsson and S. A. Riedel: Electric Circuits (Prentice Hall, New Jersey, 2015).

18 N. S. Nise: Control Systems Engineering (Addison-Wesley, New York, 1995).

19 N. S. Nise: Control Systems Engineering (John Wiley \& Sons, New York, 2008).

20 A. M. Rushdi: IEEE Trans. Education 28 (1985) 17. https://doi.org/10.1109/TE.1985.4321725

21 A. S. Sedra and K. C. Smith: Microelectronic Circuits (Oxford University Press, USA, 1998).

22 L. J. Tung and B. W. Kwan: Circuit Analysis (World Scientific, Singapore, 2001).

23 L. Zuo and S. K. Islam: IEEE Trans. Circuits Syst. I Regul. Pap. 60 (2013) 2084. https://doi.org/10.1109/ TCSI.2013.2239161 


\section{About the Authors}

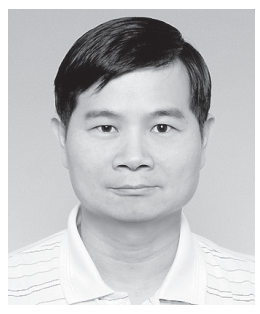

Chung-Cheng Chen received his B.S. degree from National Taiwan Normal University, Taiwan, in 1982 and his M.S. and Ph.D. degrees in electrical engineering from National Sun Yat-Sen University, Taiwan, in 1987 and 1994, respectively. He joined the Department of Electrical Engineering, National Formosa University in 1989 as a lecturer. He became a professor in 2000. In 2008, he joined the Department of Electrical Engineering, National Chiayi University. In 2011, he joined the Department of Electrical Engineering, Hwa Hsia University of Technology, as a professor, where he has been the Dean of College of Information Technology and Engineering, the CEO of the General Knowledge Committee, and Principal Secretary. He was invited to be the Dean of College of Electronic Engineering and Intelligent Manufacturing, City College of Dongguan University of Technology, China, in 2017. His research interests include biomedical engineering (HIV and cancer), neural networks, fuzzy control, nonlinear control theory and design, feedback linearization control, singularly perturbed systems and signal processing. He is currently the head of the Chen's Electric Unifying Approach R\&D team.

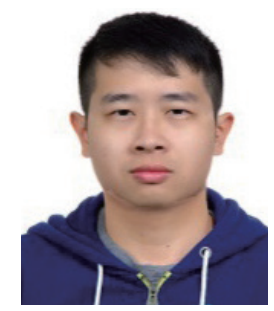

Yen-Ting Chen will graduate from Feng Chia University, Taiwan, with a B.S. degree in automatic control engineering in June 2019. He has special professional licences in audio-visual electronic, single-chip design at the practical level and web design. He has received professional awards including the Excellence Award in the 2014 Asian Robot Sports Competition among vocational high schools, first prize in the 2014 Regional Teaching Resource Center Three Innovations Competition, and second prize in the North Region Teaching Resource Center to Optimize Vocational School Competitions. He is currently a key member of the Chen's Electric Unifying Approach R\&D team.

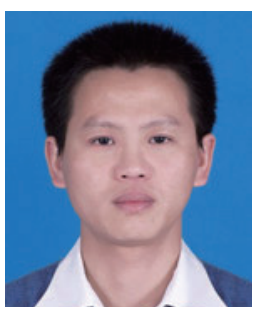

Jian-hui Li received his B.S. degree in engineering from Jiangxi University of Science and Technology and his M.S. degree in control engineering from South China University of Technology in 2005 and 2009, respectively. He joined the City College of Dongguan University of Technology as a researcher and became a senior researcher in 2018. His research interests include the application of electronic technology and wireless sensor networks. He is currently a key member of the Chen's Electric Unifying Approach R\&D team. 